\title{
Experimental Study of the Richtmyer-Meshkov Instability of Incompressible Fluids
}

\author{
BY C.E. NIEDERHAUS ${ }^{\dagger}$ AND J.W. JACOBS \\ Department of Aerospace and Mechanical Engineering, University of Arizona, \\ Tucson, AZ 85721, USA
}

The Richtmyer-Meshkov instability of a low Atwood number, miscible, two-liquid system is investigated experimentally. The initially stratified fluids are contained within a rectangular tank mounted to a sled that rides on a vertical set of rails. The instability is generated by dropping the sled onto a coil spring, producing a nearly impulsive upward acceleration. The subsequent freefall that occurs as the container travels upward and then downward on the rails allows the instability to evolve in the absence of gravity. The interface separating the two liquids initially has a well-defined, sinusoidal perturbation that quickly inverts and then grows in amplitude after undergoing the impulsive acceleration. Disturbance amplitudes are measured and compared to theoretical predictions. Linear stability theory gives excellent agreement with the measured initial growth rate, $\dot{a}_{0}$, for single-mode perturbations with the predicted amplitudes differing by less than $10 \%$ from experimental measurements up to a nondimensional time $k \dot{a}_{0} t=0.7$, where $k$ is the wavenumber. Linear stability theory also provides excellent agreement for the individual mode amplitudes of multi-mode initial perturbations up until the interface becomes multi-valued. Comparison with previously published weakly nonlinear single-mode models shows good agreement up to $k \dot{a}_{0} t=3$, while published nonlinear single-mode models provide good agreement up to $k \dot{a}_{0} t=30$. The effects of Reynolds number on the vortex core evolution and overall growth rate of the interface are also investigated. Measurements of the overall amplitude are found to be unaffected by the Reynolds number for the range of values studied here. However, experiments carried out at lower values of Reynolds numbers were found to have decreased vortex core rotation rates. In addition, an instability in the vortex cores is observed.

\footnotetext{
† Present address: NASA Glenn Research Center, 21000 Brookpark Road, Cleveland, OH 44I35, USA
} 
The time of appearance of this instability was found to increase when the Reynolds number is decreased.

\section{Introduction}

Richtmyer-Meshkov (RM, Richtmyer 1960; Meshkov 1969) instability is the instability of an impulsively accelerated planar interface separating two fluids of different density. For example, RM instability causes small perturbations on a flat interface, accelerated by a passing shock wave, to grow in amplitude and eventually become a turbulent flow. RM instability is closely related to Rayleigh-Taylor (RT, Rayleigh 1900; Taylor 1950) instability, which is the instability of a planar interface undergoing constant acceleration, such as caused by the suspension of a heavy fluid over a lighter one in the earth's gravitational field. Therefore, RM instability is often referred to as impulsive or shock-induced Rayleigh-Taylor instability. The simplicity of RM instability (in that it requires very few defining parameters), and the fact that it can be generated in a closed container, makes it an excellent fluid flow to study nonlinear stability theory as well as turbulent transport in a heterogeneous system.

RM instability is of importance to a variety of applications spanning a wide range of scales. For example, at very large scales RM instability results in mixing in supernovas. During a supernova explosion an outward propagating spherical shock wave formed in the collapsing core of a dying star passes through stratified outer gas layers of differing density producing RM instability. Observations of Supernova 1987A indicate that the helium and hydrogen outer layers experienced a significant amount of RM induced mixing as a result of this event (Arnett et al. 1989; Burrows, Hayes, \& Fryxell 1995). RM instability can also occur in high-speed combustion applications such as is in supersonic combustion ramjet engines (scramjets) in which mixing the fuel and air is a significant challenge. One proposed solution to this problem is to pass a light gaseous hydrogen fuel jet surrounded by the (heavy) air free stream through an oblique shock wave. The resulting RM instability increases the mixing, yielding an 
augmentation of the burning rate (Markstein 1957; Curran, Heiser, \& Pratt 1996). At even smaller scales, RM instability is of fundamental importance in inertial confinement fusion (ICF). ICF uses high-energy laser beams to compress a shell encapsulating a low-density deuteriumtritium fuel mixture. The shell-fuel density interface undergoes a combination of RM and RT instabilities resulting in a turbulent flow that limits the degree of compression achievable in this process. These instabilities are the most significant reason why in the experiments conducted to date the energy used to drive the lasers has greatly exceeded the energy output from the fusion process (McCall 1983; Lindl, McCrory, \& Cambell 1992; Hogan, Bangerter, \& Kulcinski 1992; Lindl 1995).

Taylor (1950) was first to use linear stability theory to analyze the growth of perturbations on a flat interface in a constant gravitational field. Using potential flow to describe the velocity field in each of the fluids, Taylor showed that the amplitude of a small sinusoidal perturbation $\eta(x, t)=a(t) \cos (k x)$ given to a system with the heavy fluid with density $\rho_{2}$ over a lighter one with density $\rho_{1}$ evolves according to the equation:

$$
a=a_{0} \cosh (\sqrt{k g A} t)
$$

where $A=\left(\rho_{2}-\rho_{1}\right) /\left(\rho_{2}+\rho_{1}\right)$ is the Atwood number and $a_{0}$ is the initial amplitude. Thus, a system oriented with a heavy fluid over a lighter one will grow exponentially in time, and the oppositely oriented configuration (light fluid over heavy) generates oscillating solutions, indicating stability. Richtmyer (1960) addressed the instability of a planar interface separating two gases that is impulsively accelerated by a planar shock wave traveling in the direction of the interface's normal. He recognized that for relatively weak shocks, this problem could be adequately modeled by considering incompressible fluids in an impulsive gravitational field. Thus, he modeled the instability using the same techniques as Taylor, except using a gravitational force in the form of a Dirac delta function $(g(t)=\Delta V \delta(t))$ and obtained the following expression for the growth rate of the perturbation: 


$$
\dot{a}=k A \Delta V a_{0}
$$

where $\Delta V$ is the velocity change imparted by the impulsive acceleration and $a_{0}$ is the initial amplitude. Thus small perturbations to the flat interface result in a constant growth rate, but, unlike the constant acceleration case, the instability occurs whether the acceleration is directed from light fluid to heavy or vice versa. When the acceleration is directed from the lighter into the heavier fluid the resulting body force has similar orientation to that producing RayleighTaylor instability. Thus the amplitude increases at a constant rate. Conversely, when the acceleration is oppositely directed the amplitude first decreases until it passes through zero, after which it emerges as a growing waveform that has been shifted in phase by $180^{\circ}$.

The linear growth stage described by Richtmyer's result lasts as long as the perturbation amplitude is sufficiently small (typically as long as $k a<1$ ). When the amplitude becomes comparable to the wavelength, the predicted growth rate decreases owing to the influence of the nonlinearity of the governing equations. The effects of weak nonlinearity can be incorporated into the stability analysis by developing a solution in the form of an asymptotic expansion using the perturbation amplitude as the small parameter (Haan 1991). These solutions, however, have the weakness that when truncated they produce results that quickly diverge from the exact solution when the amplitude reaches moderate size. Zhang \& Sohn (1997) have found a solution to this problem by posing their series solution as a Padé approximant which significantly extends its validity. One shortcoming of Zhang \& Sohn's solution is that it does not possess the generally accepted asymptotic behavior that the growth rate decays as $1 / t$ as time, $t$, approaches infinity. This weakness has been addressed by Sadot et al. (1998) who present a model that both captures the initial weakly nonlinear behavior yet also provides the correct late-time asymptotic form.

The impulsive acceleration in RM instability is typically produced by the passage of a shock wave over the interface. The most common method for generating RM instability in the laboratory is to create a boundary between two gases in a shock tube. Early shock tube RM 
experiments utilized a physical barrier to initially separate the two gases in order to prevent their mixing. However, physical barriers subsequently introduce other difficulties. The earliest of these methods, used by Meshkov (1969) and others (Aleshin et al. 1988; Vassilenko et al. 1992; Benjamin 1992), employs a sinusoidally shaped thin membrane between the two gases to separate them and provide the initial perturbation. This membrane is subsequently shattered by the incident shock wave. However, the pieces of the membrane become incorporated into the fluid flow, potentially affecting the development of the instability. The presence of the membrane fragments in the flow also impedes the visualization of the flow making techniques such as Planar Laser-Induced Fluorescence (PLIF) (Jacobs 1993), Planar Rayleigh Scattering (Budzinski, Benjamin, \& Jacobs 1994) and particle image velocimetry (Rightley et al. 1999) difficult to implement. Furthermore, this method often produces initial growth rate measurements that are significantly less than Richtmyer's theoretical prediction, typically by a factor of two or more.

Another method for interface formation employs a thin plate to separate the gases (Brouillette \& Sturtevant 1994; Cavailler et al. 1990; Bonazza \& Sturtevant 1996). When extracted prior to shock tube firing, the wake produced by the plate provides a pseudo-sinusoidal perturbation to the interface. The results of these experiments are limited by the fact that the initial perturbation is uncontrolled, nonuniform and often unrepeatable. As is characteristic of all fluid instabilities, the initial state of the system dictates future behavior, hence any variation in initial conditions makes it difficult to compare experimental results from experiment to experiment. The interfaces created by this method are also very diffuse, having thicknesses equaling or exceeding the perturbation wavelength, which significantly slows instability growth. An alternative method for forming the interface between two gases was developed by Jones \& Jacobs (1997). In these experiments the two gases flowed from opposite ends of the shock tube, exiting through slots at the initial interface location. The result was a relatively thin interface between the fluids that was sinusoidally perturbed by laterally oscillating the entire shock tube to form a standing wave. This method eliminates the influence of a membrane, yet it also provides 
repeatable initial conditions. These experiments have yielded initial growth rate measurements that are in much better agreement with linear and nonlinear theories than previous experiments.

In response to difficulties associated with the generation of interfaces between gases in shock tube experiments, a number of researchers have developed alternative experimental methods employing shock accelerated liquids or solids to produce the instability. The use of solids is particularly advantageous because of the relative ease in which a perturbation of known shape can be machined onto a solid surface. However, very strong shocks are necessary in order to make the solids behave as fluids. Benjamin \& Fritz (1987) employed a shock wave generated by an explosive charge to liquefy a layer of Wood's metal in contact with a layer of water. The sinusoidal interface machined into the Wood's metal became RM unstable, causing the perturbations to invert and then grow in amplitude. However, the interface in these experiments is also stabilized by the presence of gravity, which reduces the growth rate and makes analysis difficult. A number of investigators (Dimonte \& Remington 1993; Remington et al. 1994; Dimonte, Frerking, \& Schneider 1995; Peyser et al. 1995; Dimonte \& Schneider 1997, Farley et al. 1999, Holmes et al. 1999) have conducted experiments in which very strong shock waves are driven through targets consisting of two solids in the Nova laser facility at the Lawrence Livermore National Laboratory. In these experiments, the rapid vaporization of one end of a target produces a shock wave that travels through the density interface which has a machined initial perturbation. These experiments have demonstrated good agreement with linear and weakly nonlinear theory. However, problems associated with X-ray visualization and the difficulty in producing a pure impulsive acceleration in a laser facility make these experiments difficult to interpret.

Richtmyer (1960) recognized that RM instability need not be considered solely a compressible phenomena in that the instability can be produced by impulsively accelerating incompressible fluids. Thus a number of experimental studies have used this fact to study the "incompressible" RM instability. These experiments have the advantage in that it is relatively easy to produce a sharp well defined interface between two liquids. In addition, these 
experiments can be carried out at considerably lower speed than shock generated experiments, thus greatly simplifying flow visualization. Dimonte \& Schneider (2000) developed a Linear Electric Motor (LEM) apparatus to accelerate a container filled with two different density liquids vertically on a rail system. The LEM is capable of producing a variety of acceleration profiles, including constant and impulsive acceleration profiles which have been used to study RT and RM instabilities, respectively. The interface between the fluids was initially nominally flat, thus the primary focus of these experiments was to study the growth of the fully turbulent instability. Jacobs \& Sheeley (1996) carried out significantly lower speed RM instability experiments in which they developed a novel technique for impulsively accelerating a system of two liquids. In these experiments a Plexiglas tank containing two unequal density liquids is mounted to a linear rail system constraining its main motion to the vertical direction. The tank is gently oscillated horizontally to produce a controlled initial fluid interface shape. The sled is then released from an initial height and allowed to fall until it bounces off of a fixed spring, which imparts an impulsive acceleration in the upward direction. After bouncing, the tank travels upward and then downward on the rail system while the instability develops. Note that the effects of gravity become increasingly important as the instability growth rate is decreased. The effects of gravity are minimized in these experiments by keeping the fluids in free-fall while the instability is allowed to develop.

The experiments reported here utilize the method developed by Jacobs \& Sheeley, improved by the implementation of Planar Laser Induced Fluorescence (PLIF) imaging, yielding much clearer views of the developing interface. In addition, the experimental apparatus has been significantly improved by increasing the time duration in free-fall, direct measurement of the acceleration profile, and by allowing the generation of more complex initial perturbations. 


\section{Experimental Methods}

The experiments were conducted utilizing a 3 meter drop tower and sled with attached instrumentation, as shown in Figure 1. The drop tower's function is to provide an impulsive acceleration to the two-fluid system, and then allow the system to travel safely in free-fall without external disturbances. The tower was developed using experience gathered from the earlier apparatus of Jacobs \& Sheeley (1996). Note that the apparatus used in this study has also been used in a modified form for Rayleigh-Taylor experiments (Wadell, Niederhaus, \& Jacobs 2001). The tower consists of two pieces of vertical, 3 meter long, square steel tubing with precision linear rails mounted on the inside faces. The sled travels on the linear rails at speeds up to $6 \mathrm{~m} / \mathrm{s}$ using low-friction, high-speed bearings. A retractable spring is mounted on vertical channel behind the drop tower. The sled is able to contact the extended spring, but passes freely when the spring is fully retracted. A shock absorber at the bottom of the rails stops the sled at the end of the experiment. The sled center of mass is oriented to be centered between the linear rails and directly above the spring contact point to minimize vibrations during operation.

The two fluids are contained in a clear Plexiglas tank mounted to the sled using horizontal crossed roller bearings. The tank has interior dimensions of $254.4 \mathrm{~mm}$ high $\times 119.9 \mathrm{~mm}$ wide. Tanks having two different thicknesses were used. A $25.4 \mathrm{~mm}$ thick tank was used for the initial experiments. However, a thicker $50.8 \mathrm{~mm}$ tank was used for the majority of the experiments to minimize wall effects in the center portion of the tank. An initial sinusoidal perturbation is imposed on the density interface by gently oscillating the tank horizontally at the proper frequency to produce $n+1 / 2$ standing internal waves, where $n$ was varied from 0 to 4 . The viscous boundary layers on the side walls of the tank require the slight modification of the frequency predicted by inviscid theory. Thus, a separate experimental investigation was undertaken to determine the precise forcing frequency necessary to ensure clean, single-mode perturbations. The resulting perturbations have a measured wavelength prior to spring impact that is typically $3 \%$ greater than that predicted by inviscid theory based on tank width. 
The tank oscillation system has the capability to generate arbitrary motion. This capability allows a sinusoidal motion with the appropriate frequency and amplitude to produce a singlemode perturbation of the desired wavelength and amplitude. It also allows motion composed of a combination of different sine waves to produce more complex initial perturbations. Multimode initial perturbations are generated by oscillating the container with the superposition of the oscillations required for two or more individual modes. In both the single- and multi-mode experiments the oscillation is stopped prior to sled release at the point where the tank oscillation velocity of all modes is zero. Single mode experiments presented here are limited to modes with $21 / 2$ waves or fewer to reduce three dimensional effects that occur in higher modes in the thicker $(50.8 \mathrm{~mm})$ tank.

Planar Laser-Induced Fluorescence is used for flow visualization. A laser sheet generated from an argon-ion laser with an output of $2.8 \mathrm{~W}$ at $488 \mathrm{~nm}$ illuminates the center plane of the fluid tank. The laser sheet intensity has a Gaussian distribution and a width such that the intensity is $10 \%$ lower at the tank edges than at the center. The sheet is $4 \mathrm{~mm}$ thick at its $50 \%$ power points at the tank location. Disodium fluorescein dye is added to the heavier lower fluid at a concentration of $0.84 \mathrm{mg} / \mathrm{L}$ which fluoresces when illuminated by the laser sheet. The lighter upper fluid is clear against a black background, allowing for easy distinction of the density interface. A double-speed CCD camera mounted on the sled captures images of the fluids at a resolution of $648 \times 484$ pixels at $60 \mathrm{~Hz}$.. The images are digitized and stored in realtime by a computer video acquisition system for later analysis.

The lighter fluid used in these experiments was a water/isoproponol mixture with a $70 \%$ volume concentration of isoproponol. The heavier fluid was a water/calcium nitrate salt solution with a $25 \%$ by weight calcium nitrate concentration. These two fluids are miscible and therefore have no surface tension. Batches of fluids were mixed with sufficient volume to conduct 5 to 10 experiments with one batch. The water/calcium nitrate solution was mixed to match the index of refraction of the current batch of the water/isoproponol mixture (purchased premixed). The matching of the index of refraction was necessary to eliminate distortion of the laser sheet as it 
passes through the highly curved interface present at late experimental times. The index of refraction and specific gravity of the mixtures varied slightly from batch to batch, but a typical batch had an index of refraction of 1.3720 and a specific gravity of 0.8731 for the lighter fluid and 1.2025 for the heavier fluid. The resulting Atwood number was 0.1587 . The kinematic viscosity of the two liquids was measured for one batch using a viscometer. The lighter fluid was found to have a viscosity of $3.16 \mathrm{cSt}$, while the heavier fluid was $1.55 \mathrm{cSt}$.

To begin an experiment, the heavier bottom fluid was dispensed into the tank first to the desired level. A water-saturated piece of balsa wood slightly smaller than the interior dimensions of the tank was then placed on top of the heavy fluid. The lighter fluid was then dispensed at a slow rate immediately above the balsa wood through a small tube attached to a funnel. The balsa wood continued to float above the lighter fluid during the filling process. The total time required to add the lighter fluid, attach the lid, and begin the experiment was typically between 5 and 10 minutes. The resulting interface thickness was typically 1 pixel $(0.21 \mathrm{~mm})$ or less.

Figure 2 shows a sequence of three-dimensional renderings of the apparatus depicting an animation of a typical experiment. The sled is initially held at the top of the rails and the retractable spring mechanism is extended and locked. The tank is oscillated to produce the initial perturbation, and the sled released at the appropriate time. When the sled is released, it travels down the rails until it impacts the retractable spring and bounces upwards. As the sled travels back up the rails, the upward momentum from the recoil of the spring, together with the assistance of a bungee cord, unlocks the linkage holding the retractable spring mechanism. Gravity and the bungee assist retract the spring flush with the wall before the sled returns to the original spring location. The sled is then able to pass by the spring and travel down the rails until it impacts the shock absorber at the bottom of the rails. Thus after the initial release, the fluids experience a nearly impulsive body force in the downward direction, followed by $900 \mathrm{~ms}$ of microgravity. A piezoelectric accelerometer was used to measure the impulsive acceleration, which typically peaked at approximately $50 \mathrm{~g}\left(490 \mathrm{~m} / \mathrm{s}^{2}\right)$. A capacitive accelerometer was also 
used to measure the slight bearing drag during free-fall, which was typically less than $0.02 \mathrm{~g}(0.2$ $\mathrm{m} / \mathrm{s}^{2}$ ).

\section{Results and Discussion}

The simplest example of Richtmyer-Meshkov instability is that resulting from a small amplitude, single-mode, sinusoidal initial perturbation on a sharp interface. Figure 3 is a sequence of PLIF images showing the evolution of such an instability. This particular experiment developed from a sinusodial perturbation with a dimensionless initial amplitude of $k a_{i}=0.23$ and a wavelength $\lambda$ corresponding to $11 / 2$ waves inside the tank $(\lambda=82.6 \mathrm{~mm})$, where $k=2 \pi / \lambda$ is the perturbation wavenumber and $a_{i}$ is the perturbation initial amplitude. The first image was taken immediately before the sled impacted the spring and thus shows the initial interface shape. The impulsive acceleration in these experiments is directed from the heavier fluid into the lighter fluid, with the resulting body force on the fluids acting in the downward direction. This orientation causes the initial perturbation to invert (i.e., decrease and pass through zero) before growing in amplitude. Immediately after inversion, the interface retains a sinusoidal shape, but by image (b) the interface begins to become nonsinusodial. Vorticity is deposited along the interface by the baroclinic production mechanism during the acceleration, as given by the two-dimensional vorticity equation:

$$
\frac{D \omega}{D t}=\frac{1}{\rho} \nabla \rho \times \nabla p .
$$

where $\omega$ is the vorticity vector, which is normal to the plane of the two-dimensional motion. In this case the density gradient $\nabla \rho$ is perpendicular to the interface while the pressure gradient $\nabla p$ is hydrostatic and thus aligned with the direction of acceleration. The interaction results in a sinusoidal distribution of vorticity. However, as the instability evolves, the vorticity begins to concentrate at points midway between the crests and troughs which correspond to the points of maximum initial interface slope and thus the locations of maximum vorticity (Zabusky 1999). 
The resulting vortices produce the symmetric mushroom pattern typical of the RT and RM instability.

As time advances, the vortices appear to grow in size as the interface rotates around their centers to form a spiral pattern. The interface is multi-valued by image (c), and the vortex center has completed several turns by image (1). As the instability develops, the tips of the vortex spirals evolve into a hammerhead type form, which can be more easily observed in Figure 4 . As the vortex develops, the inner portion of the tip remains at the vortex center while the outer half is stretched in length and eventually extends over one full rotation before becoming too fine to observe. Note that the interface retains its top-to-bottom symmetry well into the nonlinear regime. This symmetry is a characteristic of the RM instability with small density differences. Also note that the interface between the two fluids also remains sharp throughout the experiment. The effects of the side walls observed in these experiments is small. A thin boundary layer is apparent on the right wall, and a small vortex forms on the left wall.

Figures 5 and 6 show sequences similar to that shown in Figure 3 with different perturbation wavelengths. Figure 5 shows the growth of the RM instability with a $1 / 2$ wave perturbation. Note that in this case the final perturbation amplitude in frame (1) is as large as the $11 / 2$ wave case of Figure 3. However, the instability itself is not as far developed as the $11 / 2$ wave case in that the interface has not yet become double-valued. This difference emphasizes the importance of the nondimensional amplitude $k a$, which is still small in the $1 / 2$ wave case. Figure 6 is a sequence of images from a perturbation with $21 / 2$ waves. One can see that in this case the features are qualitatively the same as observed in the $11 / 2$ wave case.

\subsection{Linear Growth Regime}

Richtmyer's (1960) linear stability analysis shows that the amplitude of the interface satisfies the following differential equation:

$$
\ddot{a}(t)=-k A g(t) a(t) .
$$


Note that when the gravitational acceleration is zero, as occurs when the sled is in freefall, the theoretical growth rate $\dot{a}$ is constant. Richtmyer assumed $g(t)$ to be in the form of a Dirac delta function (i.e., $g(t)=\Delta V \delta(t))$ and integrated Eq. (4) to obtain the post acceleration growth rate

$$
\dot{a}=k A \Delta V a_{0}
$$

The acceleration pulse imparted to the fluids in the present experiments has a triangular shape as shown in Figure 7, with a typical duration of $26 \mathrm{~ms}$, a peak magnitude of $50 \mathrm{~g}$, and an integrated impulse $\Delta V$ of $6.4 \mathrm{~m} / \mathrm{s}$. The length of this acceleration pulse is too long to accurately use (5) to model the early time instability growth. However, by using the measured perturbation amplitude $a$ and velocity $\dot{a}$ prior to impact, along with the measured acceleration, Eq. (4) can be numerically integrated to determine the theoretical post-impulse amplitude and velocity. Figure 8 shows the results of this integration for a typical experiment. As described above, the direction of acceleration in these experiments initially results in the temporary stabilization of the interface. Therefore, the amplitude of the crests and troughs decreases while under acceleration. However, the momentum imparted to the fluid by this action remains after the acceleration is removed. Thus the result is the inversion of the interface, and the subsequent rapid growth of the perturbation.

Figure 9 shows the early time behavior of the amplitude for all of the single-mode experiments reported here. The amplitude is nondimensionalized in this plot using the perturbation wavenumber $k$, while time is nondimensionalized using the wave number and the theoretical initial growth rate $\dot{a}_{0}$ obtained from the integration of (4). Note that $t=0$ is found by extrapolating the integrated, theoretical post-impulse perturbation growth to zero amplitude. In this nondimensionalization, linear theory has a growth rate of 1 , and is shown by the solid line in the plot. The experiments show excellent agreement with linear theory up to $k \dot{a}_{0} t=0.3$ and are within $10 \%$ of the theory at $k \dot{a}_{0} t=0.7$, where nonlinear effects begin to become important. It should be noted that linear theory is derived assuming $|k a| \ll 1$. Thus, it is surprising how 
accurate it is at moderate values of $k a$. The maximum initial amplitudes, $k a_{i}$, for the $1 / 2$ wave, 1 $1 / 2$ wave, and $21 / 2$ wave experiments were $0.06,0.50,0.66$ respectively.

\subsection{Weakly Nonlinear Growth Regime}

Figure 10 shows the intermediate-time amplitude measurements ( $\left.k \dot{a}_{0} t \leq 5\right)$, along with lines corresponding to two weakly nonlinear solutions developed by Zhang \& Sohn (1997). The first is a weakly nonlinear fourth order perturbation solution for the amplitude which was developed in much the same manner as Richtmyer's (1960) original linear analysis. Perturbation theory assumes a solution in the form of an asymptotic expansion for the surface elevation

$$
\eta=\eta^{(1)}+\eta^{(2)}+\ldots
$$

where $\eta^{(n)} \propto\left(k a_{i}\right)^{(n)}$. The solution obtained by Zhang \& Sohn assumes an impulsive body force directed from the heavy fluid into the light fluid and a finite initial amplitude. The nondimensional amplitude $k a$ can be written in terms of the parameters $k a_{i}, A$, and $\sigma=-k A \Delta V$. The solution is not directly comparable to the present experiments which have an oppositely directed as well as a finite duration acceleration. However, by letting the initial amplitude approach zero while maintaining a constant post-impulse growth rate $\left(k a_{i} \rightarrow 0\right.$ while $\sigma k a_{i}=$ constant $=k \dot{a}_{0}$ ), one can obtain an expression appropriate for comparison with our experiments. The result for the overall amplitude (the peak-to-peak amplitude divided by two) then becomes

$$
k a=k \dot{a}_{0} t-\left(\frac{1}{6}-\frac{A^{2}}{3}\right)\left(k \dot{a}_{0} t\right)^{3}+O\left(k \dot{a}_{0} t\right)^{5}
$$

Note that the second and fourth order terms do not contribute to the overall amplitude. Equation (7) is plotted in Figure 10 evaluated at a representative experimental Atwood number of 0.155. This solution agrees with the experimental data to within $10 \%$ up to $k \dot{a}_{0} t=1.3$, but then rapidly diverges due to its cubic form. 
Recognizing the limited range of validity of this type of solution, Zhang \& Sohn (1997) differentiated their amplitude perturbation expansion to develop a perturbation expansion for the growth rates of the bubble, spike, and overall amplitude. A bubble is defined as the portion of the interface where the light fluid penetrates the heavy fluid, and a spike is the portion of the interface where the heavy fluid penetrates the light fluid. The overall amplitude is the average of the bubble and spike amplitudes. These series solutions were then approximated with Padé approximants to extend their range of validity. By again taking the limit as $k a_{i} \rightarrow 0$ while keeping $\sigma k a_{i}=$ constant $=k \dot{a}_{0}$, Zhang \& Sohn's expression for the overall amplitude growth rate can be rewritten (for the case when $k a_{i}>A^{2}-\frac{1}{2}$ ) as

$$
k \dot{a}=\frac{k \dot{a}_{0}}{1+\left(\frac{1}{2}-A^{2}\right)\left(k \dot{a}_{0} t\right)^{2}},
$$

which after integration yields:

$$
k a=\frac{1}{\sqrt{\frac{1}{2}-A^{2}}} \tan ^{-1}\left(\sqrt{\frac{1}{2}-A^{2}} k \dot{a}_{0} t\right) .
$$

Evaluating (9) using $A=0.155$ results in the short dashed curve shown in Figure 10. This expression extends the range of agreement (to within $10 \%$ ) for this theory to $k \dot{a}_{0} t=3$.

\subsection{Fully Nonlinear Growth Regime}

\subsubsection{Vortex Model}

Jacobs \& Sheeley (1996) noted that the vorticity in these experiments eventually coalesces into discrete vortices. They subsequently modeled the flow as a row of line vortices of alternating sign, assuming an Atwood number of zero and obtained a relationship between the overall growth rate and amplitude that can be rearranged into the following form

$$
\frac{\dot{a}}{\dot{a}_{0}}=\frac{2}{\pi} \operatorname{sech}(k a)
$$


Figure 11 shows the derivative of the late time amplitude data $\left(k \dot{a}_{0} t \leq 30\right)$ plotted versus dimensionless amplitude along with a curve corresponding to (9a). Note that the vortex model gives an initial growth rate that is lower by a factor of $\pi / 2$ than that given by linear theory. This is consistent with the fact that in the linear stability analysis the vorticity is assumed to have a sinusoidal distribution, rather than a discrete distribution assumed by the vortex model. However, at late times, the model appears to yield excellent agreement with the experimentally observed growth rate, differing by less than $25 \%$. This small difference at late times can be attributed to the fact that this model assumes an Atwood number of 0 , while the experiments have a small but nonzero Atwood number of 0.155

Equation (9a) can be integrated to obtain an expression for the overall amplitude

$$
k a=\sinh ^{-1}\left(\frac{2}{\pi} k \dot{a}_{0}\left(t-t_{p}\right)+\sinh \left(k a_{p}\right)\right)
$$

where $t_{p}$ and $a_{p}$ are the time and amplitude, respectfully, when the vorticity is assumed to concentrate. Since $\sinh ^{-1}(x) \cong \ln (2 x)$ for large values of $x$, this model gives logarithmic late time growth. Thus the late time velocity becomes

$$
v=\frac{1}{k t}
$$

Figure 12 compares the late-time amplitude measurements ( $\left.k \dot{a}_{0} t \leq 30\right)$ with a curve generated from (10) assuming the vorticity concentrates immediately after impact, i.e. $k \dot{a}_{0} t_{p}=0$. However, since the vorticity in the experiments does not concentrate until much later the curve underestimates the amplitude. Thus, the condition $k \dot{a}_{0} t_{p}=0$ can be considered a lower bound on the amplitude. Nevertheless, the curve appears to have the same general shape as the experimental data, and differs from the measurements by less than $10 \%$ at late times. Also shown in Figure 12 is a curve generated assuming the vorticity concentrates at $k \dot{a}_{0} t_{p}=6\left(k a_{p}=\right.$ 2.4), at a point corresponding to Figure 3, frame (f) and Figure 6, frame (g). This assumption reduces the difference between the model and the late-time amplitude to less than $5 \%$ over the 
range tested. This difference may again be attributed to the fact that the model is strictly valid only for Atwood number of 0 . It is interesting to note that increasing the initial growth rate in (10) by a factor of $\pi / 2$ yields, $k a=\sinh ^{-1}\left(k \dot{a}_{0} t\right)$, which has the same functional form as (10), but also captures the correct initial growth rate. This function is also plotted in Figure 12 showing that even though it assumes an incorrect value of the circulation, it provides an excellent fit to the data.

\subsubsection{Interpolation Model}

Sadot et al. (1998) proposed the following rational function interpolation between existing early time and late time models for the bubble and spike velocities:

$$
U_{h / s}(t)=U_{0} \frac{1+B t}{1+D t+E t^{2}}
$$

where

$$
\begin{gathered}
B_{h / s}=U_{0} k, \\
D_{h / s}=(1 \pm A) U_{0} k, \\
E_{h / s}=\frac{1 \pm A}{1+A} \frac{1}{2 \pi C} U_{0}^{2} k^{2} .
\end{gathered}
$$

and $U_{0}$ is the initial velocity. In these expressions, the plus sign is used for the bubble velocity and the minus sign is used for the spike velocity. At small time the bubble and spike velocities are given by:

$$
U_{b / s}=U_{0}\left(1 \mathrm{~m} A k U_{0} t\right)
$$

which is identical to the first two terms of the fourth order weakly nonlinear perturbation solution of Zhang \& Sohn (1997). At large time the bubble and spike velocities are given by:

$$
U_{h / s}=\frac{2 \pi C(1+A)}{k(1 \pm A) t}
$$


Thus they possess the $1 / t$ dependence of the vortex model discussed above. The constant $C$ is a function of the asymptotic velocity of the bubbles and spikes. The values for $C$ given by Sadot et al. (1998) are obtained from the computations of Alon et al. (1995), in which they found C= $1 / 3 \pi$ for $A \geq 0.5$, and $1 / 2 \pi$ for $A \rightarrow 0$. However, these values can also be obtained using methods similar to those used by Takabe \& Yamamoto (1991) and Alon et al. (1995) to model the motion of an RT bubble. The differential equation governing the motion of a twodimensional bubble in a gravitational field (see Figure 13) is:

$$
\left(\rho_{1}+\kappa \rho_{2}\right) V_{b} \frac{\partial U_{b}}{\partial t}=-1 / 2 C_{D} S_{b} \rho_{2} U_{b}^{2}+\left(\rho_{2}-\rho_{1}\right) V_{b} g
$$

where $V_{b}$ is the bubble volume, $S_{b}$ is the bubble frontal area, $C_{D}$ is the bubble drag coefficient, and $\kappa$ is the virtual mass coefficient. The densities of the light and heavy fluids are $\rho_{1}$ and $\rho_{2}$, respectively. Equation (18) is a simple force balance with acceleration, drag, and buoyancy terms. In the case of RM instability in the post-impulse stage, the acceleration $g=0$. Thus, if $\kappa$ $=1$ as would be the case if the bubble had the shape of a circular cylinder, and it is assumed that $V_{b} / S_{b} \propto \lambda \propto 1 / k$, the solution for the bubble velocity is:

$$
U_{h} \propto \frac{1}{k(1+A) t}
$$

This same procedure can be used for a RM spike, employing a slightly modified form of (18), yielding:

$$
U_{s} \propto \frac{1}{k(1-A) t} .
$$

Note that Equation (11), developed using the vortex model with $A=0$, yields the asymptotic velocity $U=1 / k t$ for both the bubble and the spike. Therefore, the constant of proportionality in (19) and (20) may reasonably be assumed to be 1 for both the bubble and the spike in the small Atwood number limit. Also note that Equation (20) for the spike velocity does not apply when $A$ $=1$, in which case the drag is theoretically zero and the solution to the force balance differential 
equation (18) has a different form. Equations (19) and (20) can be used with Equation (17) (with the proportionality constant $=1$ ) to solve for $C$, yielding:

$$
C=\frac{1}{(1+A) 2 \pi}
$$

This constant $C$ equals $1 / 3 \pi$ at $A=0.5$ and $1 / 2 \pi$ at $A=0$. Thus it is in agreement with the computational results in Alon et al. (1995) in this range of Atwood numbers. However, it should be noted that (21) yields a value $C=1 / 4 \pi$ in the limit $A \rightarrow 1$ which is in disagreement with previous numerical and theoretical studies which give $C=1 / 3 \pi$ (Hecht, Alon \& Shvarts 1994; Alon et al. 1995). This difference implies that the constant of proportionality in (19) and (20) may only be 1 for small or moderate values of the Atwood number. Note that Alon et al. report using the same analytical procedure as described above but obtained different expressions for the asymptotic velocities (Equations (19) and (20)). This discrepancy was later corrected by Oron et al. (2001). However, this more recent analysis uses a much larger value for the added mass coefficient. Thus they obtain expressions for $U_{b / s}$ that differ significantly in form from ours.

The perturbation amplitude can be obtained from (12) by integrating the bubble and spike velocities separately. The result of this integration for the overall amplitude (the average of the bubble and spike amplitudes) is plotted on Figure 12 for $C=1 / 2 \pi$. The curve shows good agreement with the early-time data, but overestimates the late-time amplitude by $10 \%$. Also shown on Figure 12 is a curve generated by integrating Equation (12) using $C=1 /(1+A) 2 \pi$ with the experimental value of $A=0.155$. This curve appears to correctly model the initial growth rate, as well as the late-time asymptotic velocity. This modified form of Sadot et al.'s model shows much better agreement with the late-time amplitude measurements and appears to accurately predict the perturbation amplitude data over the entire time duration investigated. The improved agreement with the modified form of this model can be attributed to the fact that the value $C=1 / 2 \pi$ is strictly true only for Atwood number zero. Note that Alon et al. acknowledged that $C$ varied with Atwood number, but did not give a specific function for Atwood numbers less 
than $1 / 2$. It should be stressed that this model does not intrinsically solve the flow field, but is simply an interpolation that matches both the early-time and late-time growth rates.

\subsubsection{Bubble and Spike Measurements}

The overall amplitude measurements presented in the previous sections ignore differences between the bubble and spike amplitudes. As the Atwood number of the system approaches zero, the bubbles and spikes become symmetrical about the mean interface location. Thus, the bubbles and spikes have identical amplitudes, growth rates, and shapes. However, at larger Atwood numbers, differences between the bubbles and spikes become apparent. The heavier spikes grow faster than the lighter bubbles. The spikes also have smaller widths than the bubbles. In the experiments presented here with Atwood number of 0.155 , there is a small but measurable difference between the bubble and spike amplitudes.

Figure 14 shows the separate bubble and spike amplitudes for the experiments. The amplitude is measured relative to the location of the flat interface prior to impact. The growth rates of the bubbles and spikes are initially the same, as predicted by weakly nonlinear theory. However, the spike amplitude becomes $10 \%$ greater than the bubble amplitude when $k \dot{a}_{0} t=0.8$, and $30 \%$ greater when $k \dot{a}_{0} t=15$. Also shown on Figure 14 are curves corresponding to the bubble and spike amplitudes found by integrating (12). These results use our derived constant $C$ (Equation (21)) that more accurately models the late-time growth rate. Again, this modified model shows good agreement with the experimental measurements. However, experiments at other Atwood numbers would be necessary to determine whether the expression has the correct Atwood number dependence.

\subsection{Multi-Mode Initial Perturbations}

While single-mode perturbations are the most often studied in laboratory experiments, real applications involving RM instability are inevitably composed of disturbances with many 
wavelengths. Multi-mode interfacial perturbations were generated in the present investigation by oscillating the fluid container with a combination of two or more different frequencies. Figure 15 is a sequence of images showing the evolution of an experiment initiated with a combination of a $11 / 2$ wave mode and a $21 / 2$ wave mode as the initial perturbation. Image (a) was taken slightly before the impulsive acceleration and shows the initial interface shape resulting from the combination of these two modes. According to linear theory, at small amplitudes the two modes evolve independently and the mode with the shorter wavelength grows more rapidly

(since $\dot{a} \propto k a_{i}$ ). In image (b), the long wavelength mode has decreased to nearly zero amplitude, while the short wavelength mode has inverted and thus is the dominant mode visible. In images (c) through (1), this multi-mode instability evolves, forming a more complex structure than that observed in the small amplitude single-mode experiments. Note that the vorticity concentrates at points where the initial perturbation has local maxima in slope, which are also the points of maximum baroclinic vorticity generation. Also note the unusual symmetry of the interface about the center of the tank due to the small density difference of the fluids.

\subsubsection{Multi-Mode Analysis}

To analyze the multi-mode experiments, the interface was parameterized by finding the coordinates of a set of points $\left(x_{l}, y_{l}\right)$ lying on the interface using an edge detection routine, neglecting points near the sides of the tank to reduce the possible wall effects. These points were then curve-fit to a function of the form,

$$
y(x)=y_{0}+a_{1} \sin \left[k_{1}\left(x-x_{0}\right)\right]+a_{2} \sin \left[k_{2}\left(x-x_{0}\right)\right]+\ldots
$$

to determine the individual mode amplitudes, assuming $k_{1}, k_{2}, \ldots$ to be equal to that of the imposed initial perturbation. An example of the coordinates and resulting curve fit is shown in Figure 16. As mentioned above, at small amplitudes the various modes should act independently of each other and their evolution should be described by linear theory. Therefore, the method of analysis utilized for the single-mode experiments was also employed for the multi-mode 
experiments. Figure 17 shows the time dependence of the nondimensional mode amplitude for nine multi-mode experiments. The modes show excellent agreement with linear theory for $k \dot{a}_{0} t$ $<0.3$. Beyond that time the interface becomes multi-valued (at different times depending on the initial conditions) making the curve fitting of (22) no longer valid. The agreement between the measurements and theory at early times in the plot confirms that the multi-mode perturbations do act independently and follow linear theory in the small amplitude regime.

\subsubsection{Multi-Mode Examples}

Other examples of multi-mode RM instabilities are shown in Figures 18 through 20. Figure 18 shows the RM instability resulting from the combination of a $1 / 2$ wave and $21 / 2$ wave initial perturbation. The two different wavelengths are clearly present in image (a), taken before the impulsive acceleration. This initial perturbation produces three vortices of the same sign with the center vortex larger in size. The development of an initial perturbation with $11 / 2$ waves and $41 / 2$ waves is shown in Figure 19. Note that the $41 / 2$ wave mode is the second harmonic of the $11 / 2$ wave mode. Thus it produces two vortices per fundamental half wavelength in contrast to one vortex per half wavelength produced the single-mode experiments. This mode combination evolves to form a double-mushroom shape with vertically stacked vortices. The detailed development of this and all multi-mode experiments depended strongly on the relative amplitudes of the initial modes.

Figure 20 shows the RM instability produced by a $1 / 2$ wave and $41 / 2$ wave initial perturbation. Five vortices of the same sign are formed over the half wavelength of the fundamental perturbation. The central three vortices appear to be the same strength, thus this flow closely resembles Kelvin-Helmholtz instability. Image (a) shows that the amplitude of the $1 / 2$ wave perturbation is much larger than that of the $41 / 2$ wave perturbation. The large $1 / 2$ wave perturbation produces a shear flow across the tank, with the small $41 / 2$ wave perturbation serving as an initial perturbation for the Kelvin-Helmholtz instability. 


\subsection{Reynolds Number Effects}

\subsubsection{Reynolds Number Definition}

An important aspect of RM instability that has not been discussed in earlier investigations is the dependence of the Reynolds number on the flow. The Reynolds number is defined as $R e=U l / v$, where $U, l$, and $v$ are characteristic values of velocity, length, and kinematic viscosity. Obvious length and velocity scales appropriate in RM instability are the perturbation amplitude $a$ and growth rate $\dot{a}$, yielding

$$
R e_{p}=\frac{a \dot{a}}{\left(v_{1}+v_{2}\right) / 2}
$$

Figure 21 shows a plot of Reynolds number defined using (23) for a typical single-mode experiment from this investigation which shows that (23) yields a time dependent value. Thus, defined this way, the Reynolds number initially grows linearly in time (as is indicated by linear theory) peaking at $k \dot{a}_{0} t=1.6$ and then decays toward zero. It is important to recognize that the flow at late time is dominated by the vortices that form at the nodes of the perturbation. Thus, an alternative Reynolds number more appropriate to the vortical flow may be defined using the circulation of one of the vortices and the average kinematic viscosity of the two fluids:

$$
\operatorname{Re}=\frac{\Gamma}{\left(v_{1}+v_{2}\right) / 2} .
$$

Note that the circulation cannot be easily measured in the present experiments. However, it can be estimated from measurements of the initial growth rate as was done by Jacobs \& Sheeley (1996). If one assumes the flow field to be that given linear stability theory, the circulation can be found by integrating the vortex sheet strength over one-half wavelength of the interface. The result is that the circulation strength of a vortex is given by:

$$
\Gamma=\frac{4 \dot{a}_{0}}{k},
$$


which is constant during the experiment if viscous dissipation and the baroclinic generation of secondary vorticity is negligible. Therefore, the vortex Reynolds number can be calculated using:

$$
R \boldsymbol{e}=\frac{8 \dot{a}_{0}}{k\left(v_{1}+v_{2}\right)}
$$

Thus this definition yields a Reynolds number that has a constant value. This vortex Reynolds number $\left(R e_{v}\right)$ can be related to the perturbation Reynolds number $\left(R e_{p}\right)$. Figures 9,11 , and 12 demonstrate that the dimensionless amplitude $k a$ in the experiments is a function of only the dimensionless time scale $k \dot{a}_{0} t$, i.e.

$$
k a=f\left(k \dot{a}_{0} t\right)
$$

therefore

$$
k \dot{a}=k \dot{a}_{0} f^{\prime}\left(k \dot{a}_{0} t\right),
$$

and the perturbation Reynolds number can be written as

$$
\begin{aligned}
\operatorname{Re}_{p} & =\frac{a \dot{a}}{\left(v_{1}+v_{2}\right) / 2} \\
& =\frac{1}{k^{2}} \frac{2 k a k \dot{a}}{v_{1}+v_{2}} \\
& =\frac{2 \dot{a}_{0}}{k\left(v_{1}+v_{2}\right)} f f^{\prime} \\
& =\operatorname{Re}_{v} \frac{f f^{\prime}}{4}
\end{aligned}
$$

Therefore, the perturbation Reynolds number is equal to the vortex Reynolds number multiplied by a function of time, the value of which ranges from 0 to 0.16 . This vortex Reynolds number is also proportional to the Reynolds number defined using initial growth rate and perturbation wavelength as velocity and length scales. Unless specifically noted, values of the Reynolds number quoted in the following text refer to the vortex Reynolds number (24). Note that the 
value of Reynolds number achievable in an experiment varies greatly with the perturbation wavelength. Thus it is possible to achieve much larger values of circulation and Reynolds number using longer wavelength perturbations. The Reynolds number for the experiments presented here range from 1000 to 8500 .

\subsubsection{Vortex Turning Rate}

The experiments in this study spanned a wide range of initial amplitudes and Reynolds numbers. It is therefore remarkable that the nondimensional scaling used in Figures 9, 11, and 12 effectively collapses the amplitude measurements for the entire range experimental parameters investigated. This degree of collapse indicates that the late time amplitude is determined only by the perturbation wavenumber and initial growth rate. Thus, the Reynolds number does not appear to influence the amplitude measurements. However, the Reynolds number was observed in the experiments to have an effect on the evolution of the vortex cores. This effect became apparent when examining the experimental images to quantify the vortex core turning rate (i.e. the rate of rotation of the vortex cores). One method of quantifying the turning rate is to determine the nondimensional time when the interface becomes multi-valued and when the center of the vortex completes a specified number of turns. Figure 22 shows measurements of the time when the interface first becomes multi-valued and when the vortex core has completed 1,2, and 3 turns plotted as a function of the Reynolds number. The interface is considered multi-valued when its maximum slope becomes infinite, i.e. when it has rotated $90^{\circ}$ from a horizontal position. Defining the number of turns is more difficult and subjective in part because of the non-symmetrical form and rapid rate of the initial vortex development. After attaining infinite slope, the interface in a small region near the vortex core rapidly rotates $180^{\circ}$ and develops a fold, that forms the tip of the coil that eventually becomes the vortex spiral. For consistency, this folded condition is considered to be the starting point in the rotation process. Turning is then defined as the angular rotation of this heavy (dyed) fluid tip. A completed turn is defined as when the heavy fluid tip of the spiral is vertical and has rotated $n \times 360^{\circ}$ from the 
initial folded condition. Referring back to Figure 3, the interface is nearly vertical in image (b), and the vortex core has completed slightly more than 1 turn in image (e), and slightly more than 2 turns in image (h). The vortex core shown earlier in Figure 4 has completed slightly over 2 turns. Note that this definition yields a nearly constant time between turns and thus a constant rotation rate.

Figure 22 shows that the interface becomes multi-valued at $k \dot{a}_{0} t \cong 1.5$ for all the experiments in the Reynolds number range tested for both the $11 / 2$ and $21 / 2$ wave cases. The time to complete 1,2, and 3 turns appears to be independent of the Reynolds number for experiments with Reynolds numbers greater than 6000 over the times investigated. Therefore, the flow appears to be inertia dominated above a Reynolds number of 6000 . At lower Reynolds number, the turning rate is observed to decrease as the Reynolds number is decreased indicating that the fluid viscosity is important. Thus the primary effect of viscosity in these experiments is to reduce the number of coils observed in the vortex cores. Viscosity causes the vorticity to diffuse out from the vortex centers, leading to a decrease in the turning rate of the vortex cores. This process will eventually cause the vorticity from adjacent vortices to merge reducing the circulation, and thus, the overall growth rate. The fact that the amplitude measurements in this study appear to be independent of the Reynolds number suggests that vortex core sizes must still be small when compared to the vortex spacing for these experiments.

Note that the rotation rate measurements are very consistent for experiments carried out with a particular perturbation wavelength. However, there appears to be an inconsistency between experiments carried out with different wavelengths. This inconsistency is most easily observed in Figure 22 when comparing the time to attain 3 turns for the $11 / 2$ and $21 / 2$ wave experiments. This discrepancy may have been caused by the fact that the dimensionless initial amplitudes of the shorter wavelength experiments were generally larger than their longer wavelength counterparts in order to obtain the same value of Reynolds number. In other words, a $21 / 2$ wave experiments must have larger dimensionless initial amplitude in order to achieve the same Reynolds number as a $11 / 2$ wave experiments with the same impulsive acceleration. Thus the 
effects of nonlinearity may have produced the small differences in the tuning rate observed in Figure 22.

\subsubsection{Vortex Instability}

Experiments carried out early in this investigation with relatively low values of the Reynolds number consistently showed a laminar spiraling of the interface around the vortex center. However, later experiments carried out with significantly larger initial amplitudes and thus larger Reynolds numbers showed the appearance of an apparent secondary instability inside the vortex cores. Figure 23 shows a series of images with $k a_{i}=0.29$ and a Reynolds number of 4830 exhibiting this behavior. The instability initially develops very similarly to the lower amplitude cases. However, at frame (h), one can see the beginning of a secondary instability in the core of the vortex. By frame $(\mathrm{k})$ the instability has spread throughout the core and the interface is no longer sharp, indicating that the fluids have begun to mix on a smaller scale. Thus it appears that this is the beginnings of the transition to turbulence of the vortex cores.

Figure 24 shows a close-up of the core of an unstable vortex. The secondary instability initially takes the form of waves superimposed on the core spiral. These waves initiate near the center of the core and grow in size and extent until all layers of the core spiral are effected. The nondimensional time when the amplitude of these secondary instability waves equals the spiral thickness is shown plotted in Figure 25 versus the Reynolds number. Also shown is the dimensionless duration of the experiments in which the vortices remained stable throughout the experiment. One can clearly see that the experiments with larger Reynolds numbers develop the secondary instability sooner. Furthermore, this transition time appears to be well correlated to when the core has made approximately three complete turns. 


\section{Conclusions}

Incompressible Richtmyer-Meshkov instability is studied in a novel experimental apparatus that allows for a quantitative analysis of the two-dimensional instability from the early linear stages, through the nonlinear regime, and into the initial stages of the transition to turbulence. Miscible liquids with moderate Atwood number were employed in this investigation. The use of liquids avoided many of the experimental difficulties previously limiting the study of the RM instability. The instability was generated by elastically bouncing a fluid-filled container off a vertical spring, imparting an impulsive acceleration. The subsequent free-fall permitted the instability to evolve in the absence of gravity far into the nonlinear stages. Planar Laser-Induced Fluorescence allowed for extremely clear observations of the flow through the nonlinear regime.

Amplitude measurements are found to be in excellent agreement with linear stability theory for small amplitudes, differing by less than $10 \%$ up to a nondimensional time $k \dot{a}_{0} t$ of 0.7 . Linear stability theory also shows excellent agreement for multi-mode experiments until the interface became multi-valued. The fourth order, single-mode perturbation solution of Zhang \& Sohn (1997) is found to provide good agreement with amplitude measurements up to $k \dot{a}_{0} t$ of 1.3 . Zhang \& Sohn's Padé approximation to their perturbation solution is found to extend the range of agreement for amplitude up to $k \dot{a}_{0} t$ of 3 . A discrete vortex model (Jacobs \& Sheeley 1996) and the model of Sadot et al. (1998) are also compared to experimental amplitudes in the nonlinear regime. These two models were are shown to be within $10 \%$ of the amplitude measurements up to $k \dot{a}_{0} t$ of 30 . The best agreement between experiments and theory is obtained from Sadot et al.'s model modified by using a new, estimate for the asymptotic velocity.

Previous studies have not considered the influence of Reynolds number on the RM instability. This study examines the effects of Reynolds number defined using the vortex circulation. The time dependence of the overall perturbation amplitude is found to be independent of Reynolds number. However, the evolution of the vortex cores is found to be influenced by the Reynolds number when its value is sufficiently low. For the time duration 
studied, experiments with Reynolds numbers greater than 6000 are found to be independent of Reynolds number, indicating the flow is inertia dominated. Decreasing Reynolds number is found to lower the vortex turning rate. A secondary instability is observed to occur in the vortex cores of experiments with higher values of the Reynolds number. Measurements of the time when the secondary instability was first manifest appear to correlate well with the Reynolds number and is observed to occur when the core has made approximately three complete turns.

This research was supported by Lawrence Livermore National Laboratory and by NASA's Microgravity Fluid Physics Program. 


\section{REFERENCES}

Aleshin, A. N., Gamalii, E. G., Zaitsev, S. G., Lazareva, E. V., Lebo, I. G. \& Rozanov, V. B. 1988 Nonlinear and transitional states in the onset of the Richtmyer-Meshkov instability. Sov. Tech. Phys. Lett. 14, 466-468 [Russian: Pis'ma Zh. Tekh. Fiz. 14, 1063-1067].

Alon, U., Hecht, J., Ofer, D., \& Shvarts, D. 1995 Power Laws and Similarity of Rayleigh-Taylor and Richtmyer-Meshkov Mixing Fronts at All Density Ratios. Physical Review Letters 74, 534-537.

Arnett, W. D., Bahcall, J. N., Kirshner, R. P., \& Woosley, S. E. 1989 Supernova 1987A. Annual Review of Astronomy and Astrophysics 27, 629-700.

Benjamin, R. F. 1992 Experimental observations of shock stability and shock-induced turbulence. In Advances in Compressible Turbulent Mixing, Proceedings of the First International Workshop on the Physics of Compressible Turbulent Mixing, Edited by W. P. Dannevik, A. C. Buckingham, C. E. Leith, Published by Lawrence Livermore National Laboratory, Conf-8810234, 341-348.

Benjamin, R. F., \& Fritz J. N. 1987 Shock loading a rippled interface between liquids of differenct densities. Physics of Fluids 30, 331-336.

Bonazza, R., \& Sturtevant, B. 1996 X-ray measurements of growth rates at a gas interface accelerated by shock waves. Physics of Fluids 8, 2496-2512.

Brouillette, M., \& Sturtevant, B. 1994 Experiments on the Richtymyer-Meshkov instability: single-scale perturbations on a continuous interface. Journal of Fluid Mechanics 263, 271 292.

Budzinski, J. M., Benjamin, R. F., \& Jacobs, J. W. 1994 Influence of initial conditions on the flow patterns of a shock-accelerated thin fluid layer. Physics of Fluids 6, 3510-3512.

Burrows, A., Hayes, J., \& Fryxell, B. A. 1995 On the nature of core-collapse supernova explosions. The Astrophysical Journal 450, 830-850.

Cavailler, C., Mercier, P., Rodriguez, G. \& Haas, J. F. 1990 A new vertical shock tube for Rayleigh-Taylor instability measurements. Proceedings of the $17^{\text {th }}$ ISSWST at Bethlehem PA; Current Topics in Shock Waves, Y. Kim editor, 564.

Curran, E., Heiser, W., \& Pratt, D. 1996 Fluid phenomena in scramjet combustion systems. Annual Review of Fluid Mechanics 28, 323-360.

Dimonte, G., \& Remington, B. 1993 Richtmyer-Meshkov Experiments on the Nova Laser at High Compression. Physical Review Letters 70, 1806-1809.

Dimonte, G., Frerking, C. E., \& Schneider, M. 1995 Richtmyer-Meshkov Instability in the Turbulent Regime. Physical Review Letters 74, 4855-4858.

Dimonte, G., \& Schneider, M. 1997 Turbulent Richtmyer-Meshkov instability experiments with strong radiatively driven shocks. Physics of Plasmas 4, 4347-4357. 
Dimonte, G., \& Schneider, M. 2000 Density ratio dependence of Rayleigh-Taylor mixing for sustained and impulsive acceleration histories. Physics of Fluids 12, 304-321.

Farley, D. R., Peyser, T. A., Logory, L. M., Murray, S. D., \& Burke, E. W. 1999 High Mach number mix instability experiments of an unstable density interface using a single-mode, nonlinear initial perturbation. Physics of Plasmas 6, 4304-4317.

Haan, S. W. 1991 Weakly nonlinear hydrodynamic instabilities in inertial fusion. Physics of Fluids B 3, 2349-2355.

Hecht, J., Alon, U., \& Shvarts, D. 1994 Potential flow models of Rayleigh-Taylor and Richtmyer-Meshkov bubble fronts. Phys Fluids 6, 4019-4030.

Hogan, W. J., Bangerter, R., \& Kulcinski, G. L. 1992 Energy from inertial fusion. Physics Today $45,42-50$.

Holmes, R. L., Dimonte, G., Fryxell, B., Gittings, M. L., Grove, J. W., Schnieder, M., Sharp, D. H., Velikovich, A. L., Weaver, R. P., \& Zhang, Q. 1999 Richtmyer-Meshkov instabiity growth: experiment, simulation, and theory. Journal of Fluid Mechanics 389, 55-79.

Jacobs, J. W. 1993 The dynamics of shock accelerated light and heavy gas cylinders. Physics of Fluids A 5, 2239-2247.

Jacobs, J. W., \& Sheeley, J. M. 1996 Experimental study of incompressible Richtmyer-Meshkov instability. Physics of Fluids 8, 405-415.

Jones, M. A., \& Jacobs, J. W. 1997 A membraneless experiment for the study of RichtmyerMeshkov instability of a shock-accelerated gas interface. Physics of Fluids 9, 3078-3085.

Lindl, J. D., McCrory, R. L., \& Campbell, E. M. 1992 Progress toward ignition and burn propagation in inertial confinement fusion. Physics Today 45, 32-40.

Lindl, J. D. 1995 Development of the indirect-drive approach to inertial confinement fusion and the target physics basis for ignition and gain. Physics of Plasmas 2, 3933-4024.

Markstein, G. H. 1957 A shock tube study of flame front-pressure wave interaction. In 6th International Symposium on Combustion, Reinhold Publications Corporation, 387-398.

McCall, G. H. 1983 Laser-driven implosion experiments. Plasma Physics 25, 237-285.

Meshkov, E. E. 1969 Instability of the interface of two gases accelerated by a shock wave. Izvestiia Akademii Nauk SSSR Mekhanika Zhidkosti i Gaza 4, 151-157.

Oron, D., Arazi, L., Kartoon, D, Rikanati, A., Alon, U., \& Shvarts, D. 2001 Dimensionality dependence of the Rayleigh-Taylor and Richtmyer-Meshkov instability late-time scaling laws. Physics of Plasmas 8, 2883-2889.

Peyser, T. A., Miller, P. L., Stry, P. E., Budil, K. S., Burke, E. W., Wojtowicz, D. A., Griswold, D. L., Hammel, B. A., \& Phillion, D. W. 1995 Measurement of Radiation-Driven ShockInduced Mixing from Nonlinear Initial Perturbations. Physical Review Letters 75, 23322335. 
Rayleigh, Lord 1900 The Scientific Papers of Lord Rayleigh, Vol. II, Cambridge University Press.

Remington, B. A., Weber, S. V., Marinak, M. M., Haan, S. W., Kilkenny, J. D., Wallace, R., \& Dimonte, G. 1994 Multimode Rayleigh-Taylor Experiments on Nova. Physical Review Letters 73, 545-548.

Richtmyer, R. D. 1960 Taylor Instability in Shock Acceleration of Compressible Fluids. Communications on Pure and Applied Mathematics 13, 297-319.

Rightley, P. M., Vorobieff, P., Martin, R., \& Benjamin, R. F. 1999 Experimental observations of the mixing transition in a shock-accelerated gas curtain. Physics of Fluids 11, 186-200.

Sadot, O., Erez, L., Alon, U., Levin, L. A., Erex, G., Ben-Dor, G., \& Shvarts, D. 1998 Study of Nonlinear Evolution of Single-Mode and Two-Bubble Interaction under Richtmyer-Meshkov Instability. Physical Review Letters 80, 1654-1657.

Takabe, H., \& Yamamoto, A. 1991 Reduction of turbulent mixing at the ablation front of fusion targets. Physical Review A 44, 5142-5149.

Taylor, G. I. 1950 The instability of liquid surfaces when accelerated in a direction perpendicular to their planes. Proceedings of the Royal Society of London Series A 201, 192-196.

Vassilenko, A. M., Buryakov, O. V., Kuropatenko, V. F., Olkhovskaya, V. I., Ratnikov, V. P. \& Jakovlev, V. G. 1992 Experimental research of gravitational instability and turbulization of flow at the noble gases interface. In Advances in Compressible Turbulent Mixing, First International Workshop on the Physics of Compressible Turbulent Mixing, Edited by Dannevik, W. P., Buckingham, A. C., Leith, C. E., Published by Lawrence Livermore National Laboratory, Conf-8810234, 581-606.

Waddell, J. T., Niederhaus, C. E., \& Jacobs, J. W. 2001 Experimental study of Rayleigh-Taylor instability: Low Atwood number liquid systems with single-mode initial perturbations. Physics of Fluids 13, 1263-1273.

Zabusky, N. J. 1999 Vortex Paradigm for accelerated inhomogeneous flows: Visiometrics for the Rayleigh-Taylor and Richtmyer-Meshkov environments. Annual Review of Fluid Mechanics 31, 495-536.

Zhang, Q., \& Sohn, S. 1997 Nonlinear theory of unstable fluid mixing driven by shock wave. Physics of Fluids 9, 1106-1124. 


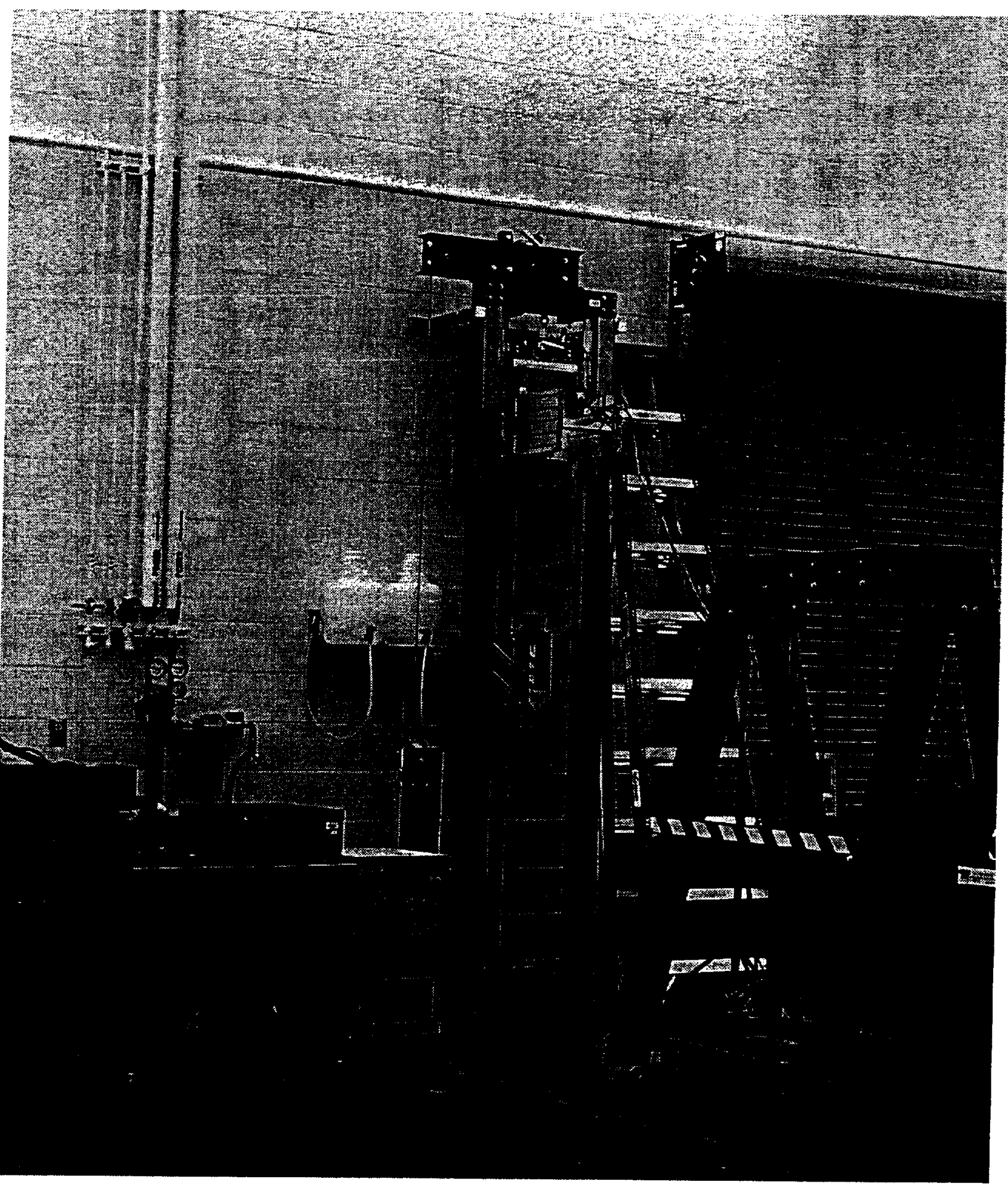

FIGURE 1. Close-up picture of the 3 meter drop tower with the sled at the top. 


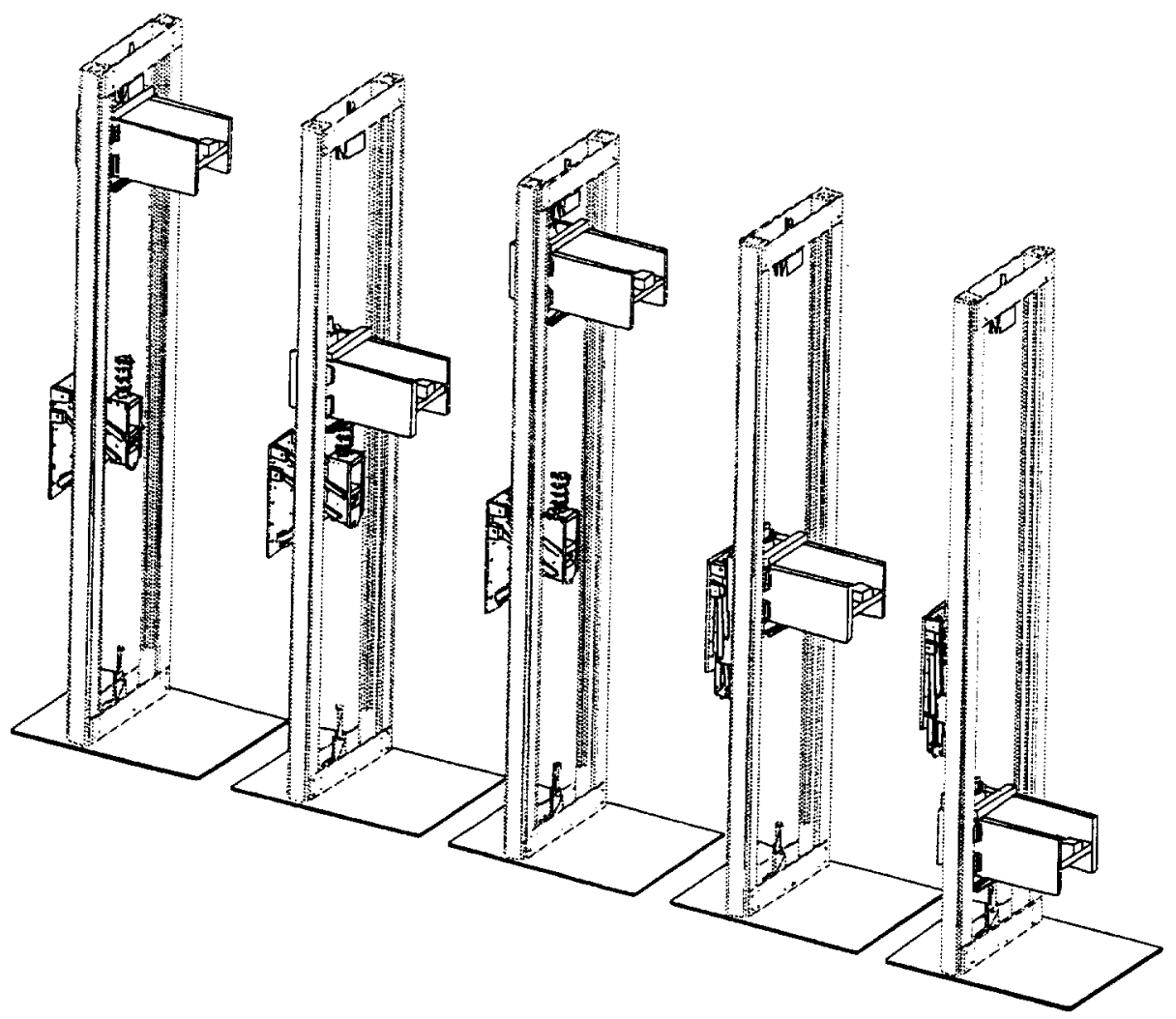

FIGURE 2. A sequence of images showing the sled traveling on the rail system during a typical experiment. 

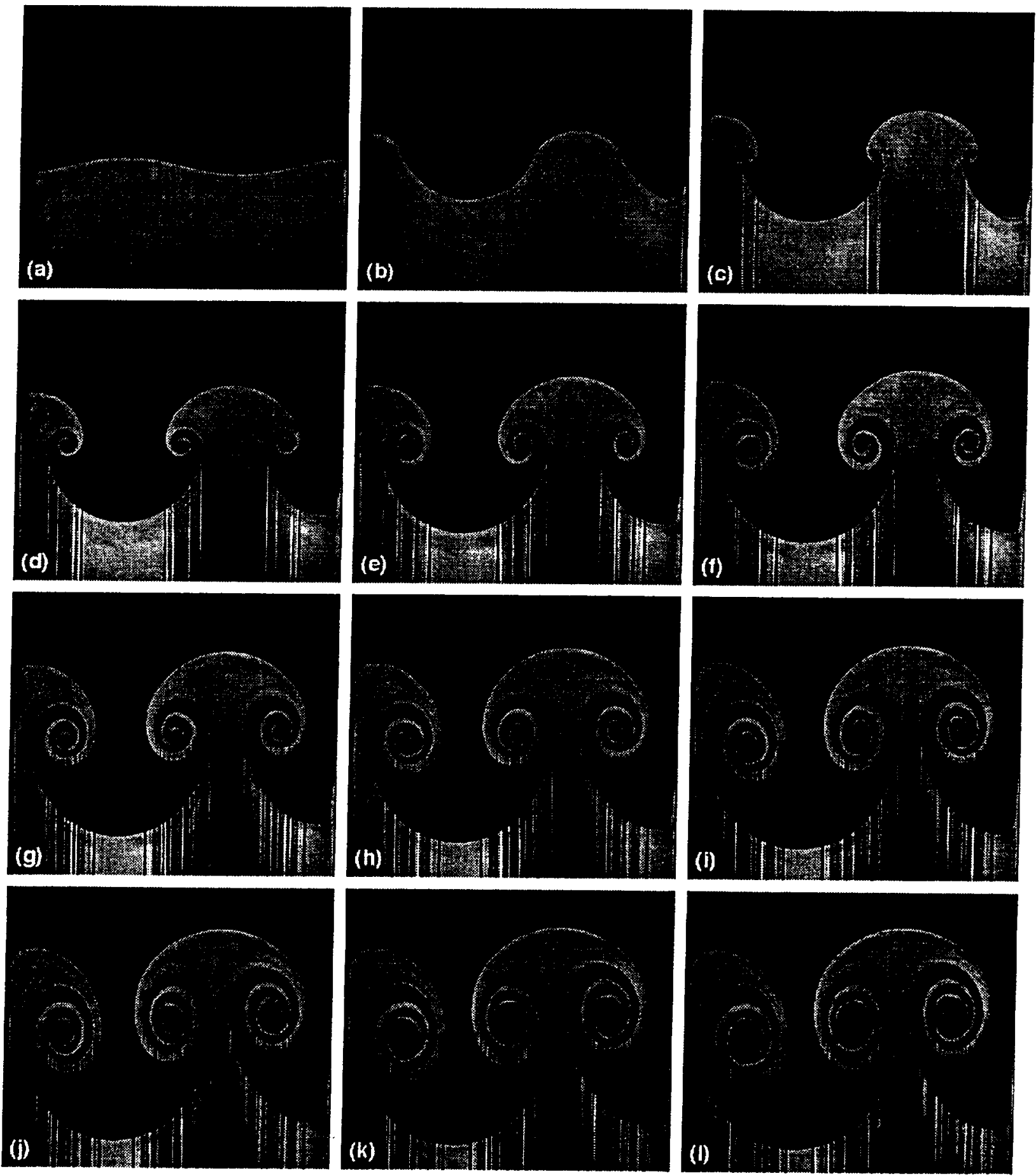

FIGURE 3. A sequence of images from an experiment with $11 / 2$ waves and $k a_{i}=0.23$. Times relative to the midpoint of spring impact are (a) $-14 \mathrm{~ms}$, (b) $102 \mathrm{~ms}$, (c) $186 \mathrm{~ms}$, (d) $269 \mathrm{~ms}$, (e) $353 \mathrm{~ms}$, (f) $436 \mathrm{~ms}$, (g) $520 \mathrm{~ms}$, (h) $603 \mathrm{~ms}$, (i) $686 \mathrm{~ms}$, (j) $770 \mathrm{~ms}$, (k) $853 \mathrm{~ms}$, and (l) $903 \mathrm{~ms}$. 


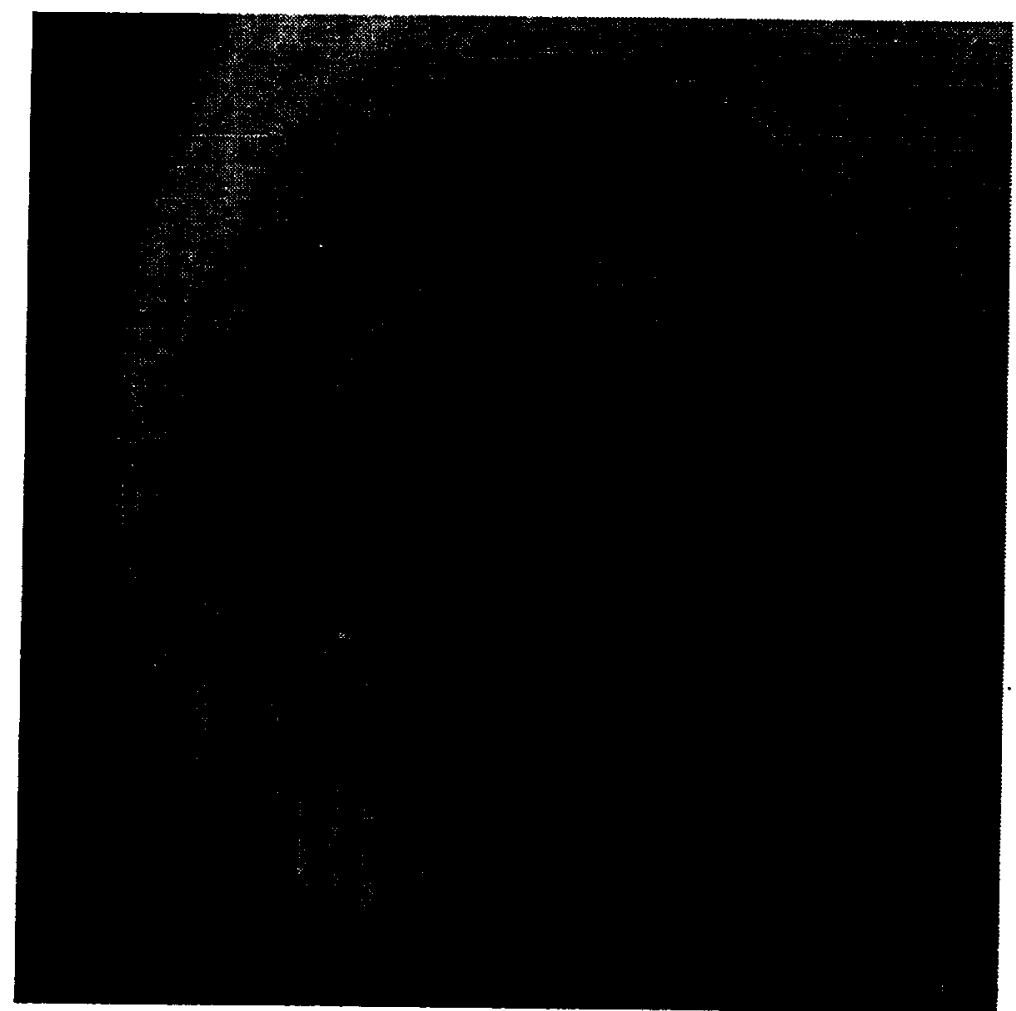

FIGURE 4. A close-up of a vortex core showing the distortion of the vortex tip. 

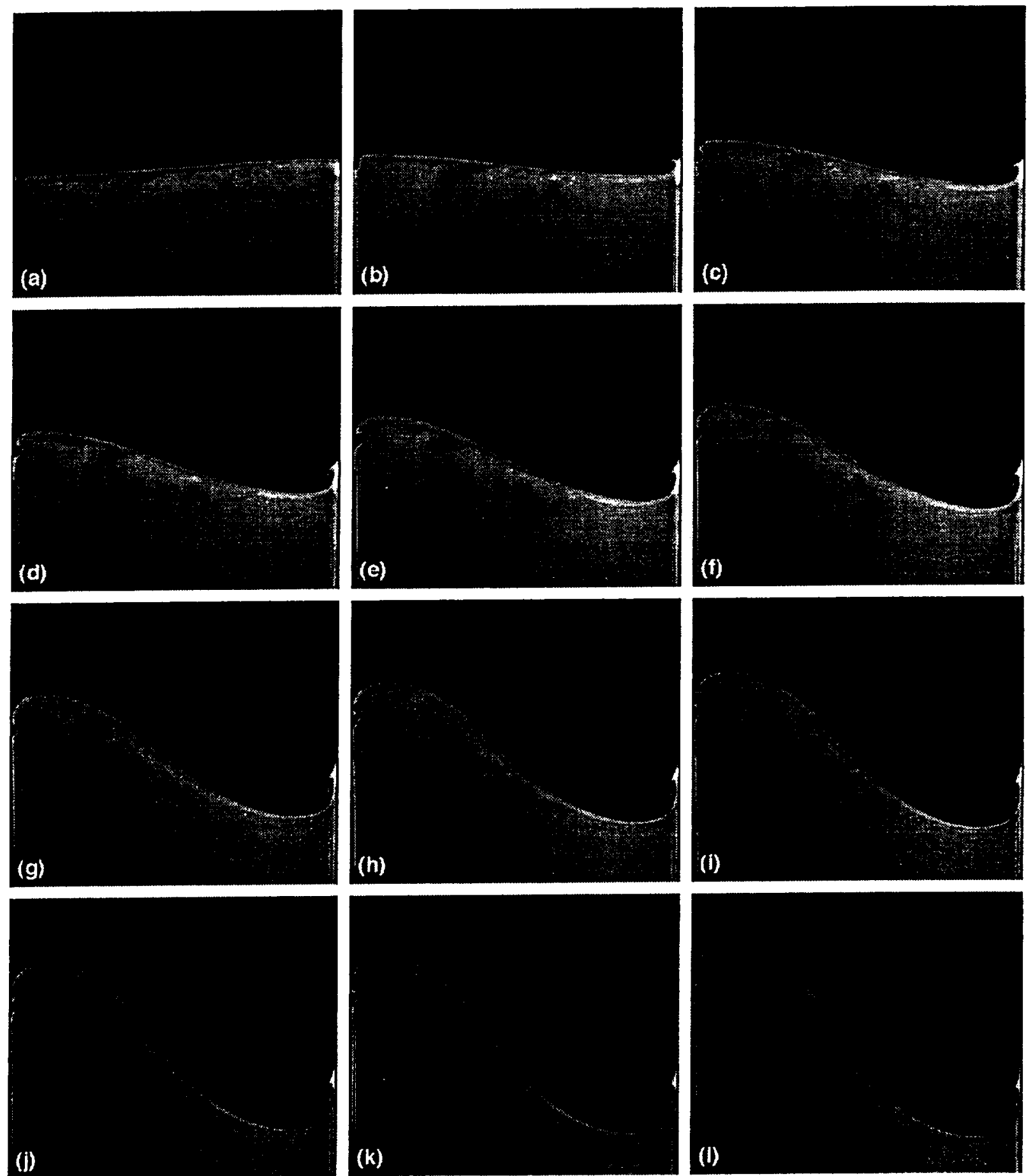

FIGURE 5. A sequence of images from an experiment with a $1 / 2$ wave and $k a_{i}=0.06$. Times relative to the midpoint of spring impact are (a) $-13 \mathrm{~ms}$, (b) $120 \mathrm{~ms}$, (c) $204 \mathrm{~ms}$, (d) $270 \mathrm{~ms}$, (e) $354 \mathrm{~ms}$, (f) $437 \mathrm{~ms}$, (g) $504 \mathrm{~ms}$, (h) $587 \mathrm{~ms}$, (i) $671 \mathrm{~ms}$, (j) $738 \mathrm{~ms}$, (k) $821 \mathrm{~ms}$, and (l) $904 \mathrm{~ms}$. 

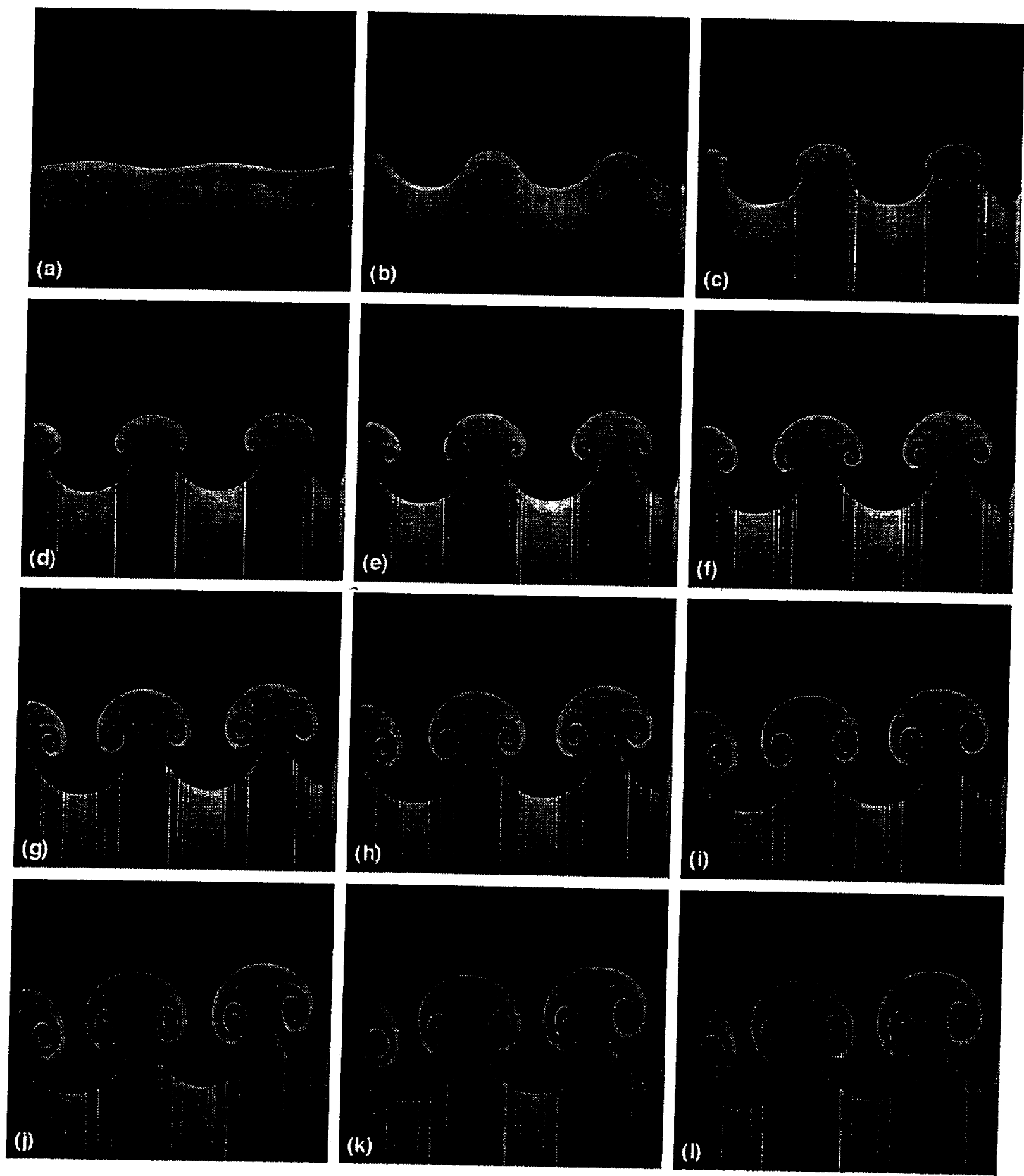

FIGURE 6. A sequence of images from an experiment with $21 / 2$ waves and $k a_{i}=0.16$. Times relative to the midpoint of spring impact are (a) $-25 \mathrm{~ms}$, (b) $92 \mathrm{~ms}$, (c) $175 \mathrm{~ms}$, (d) $259 \mathrm{~ms}$, (e) $342 \mathrm{~ms}$, (f) $426 \mathrm{~ms}$, (g) $509 \mathrm{~ms}$, (h) $592 \mathrm{~ms}$, (i) $676 \mathrm{~ms}$, (j) $759 \mathrm{~ms}$, (k) $843 \mathrm{~ms}$, and (l) $909 \mathrm{~ms}$. 


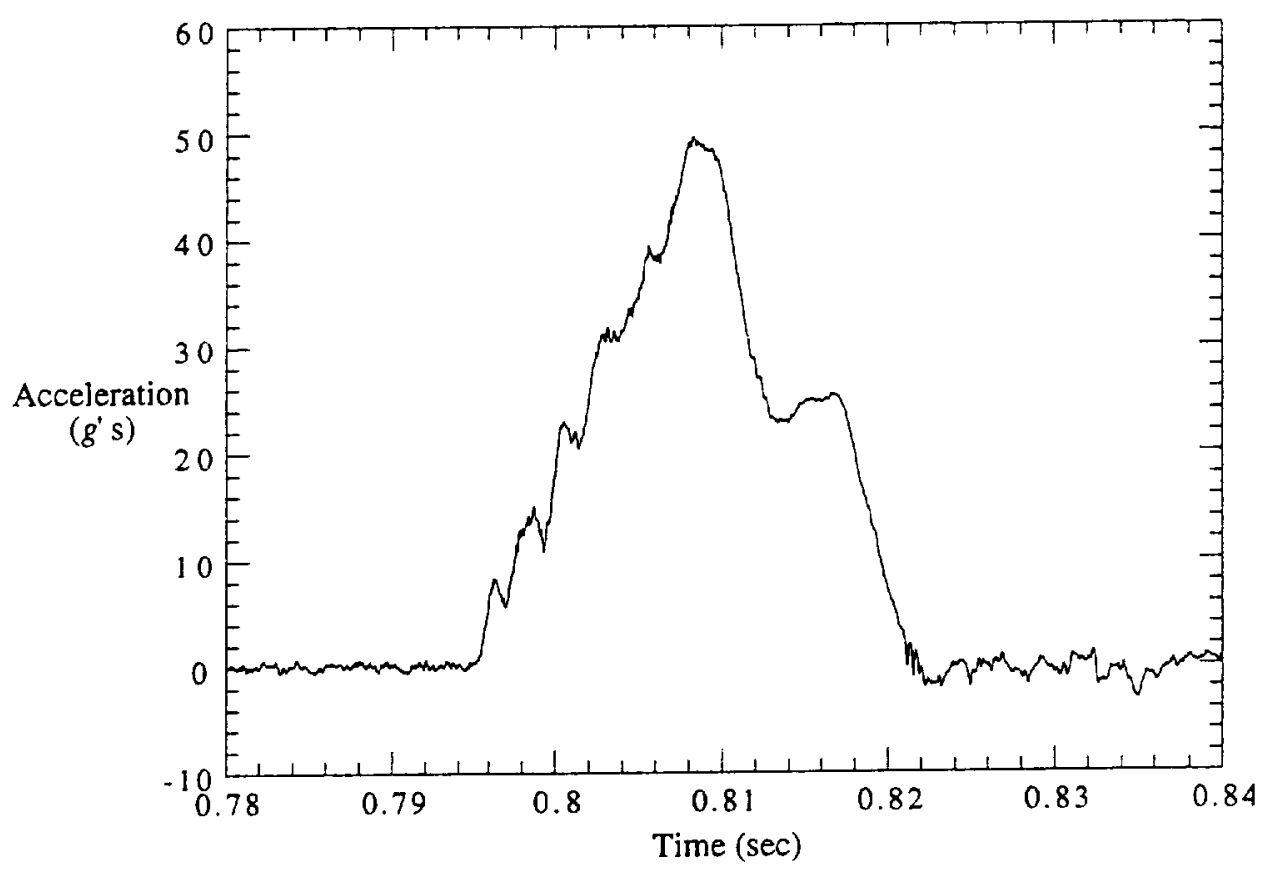

FIGURE 7. Plot of the acceleration of the system versus time for a typical experiment. 


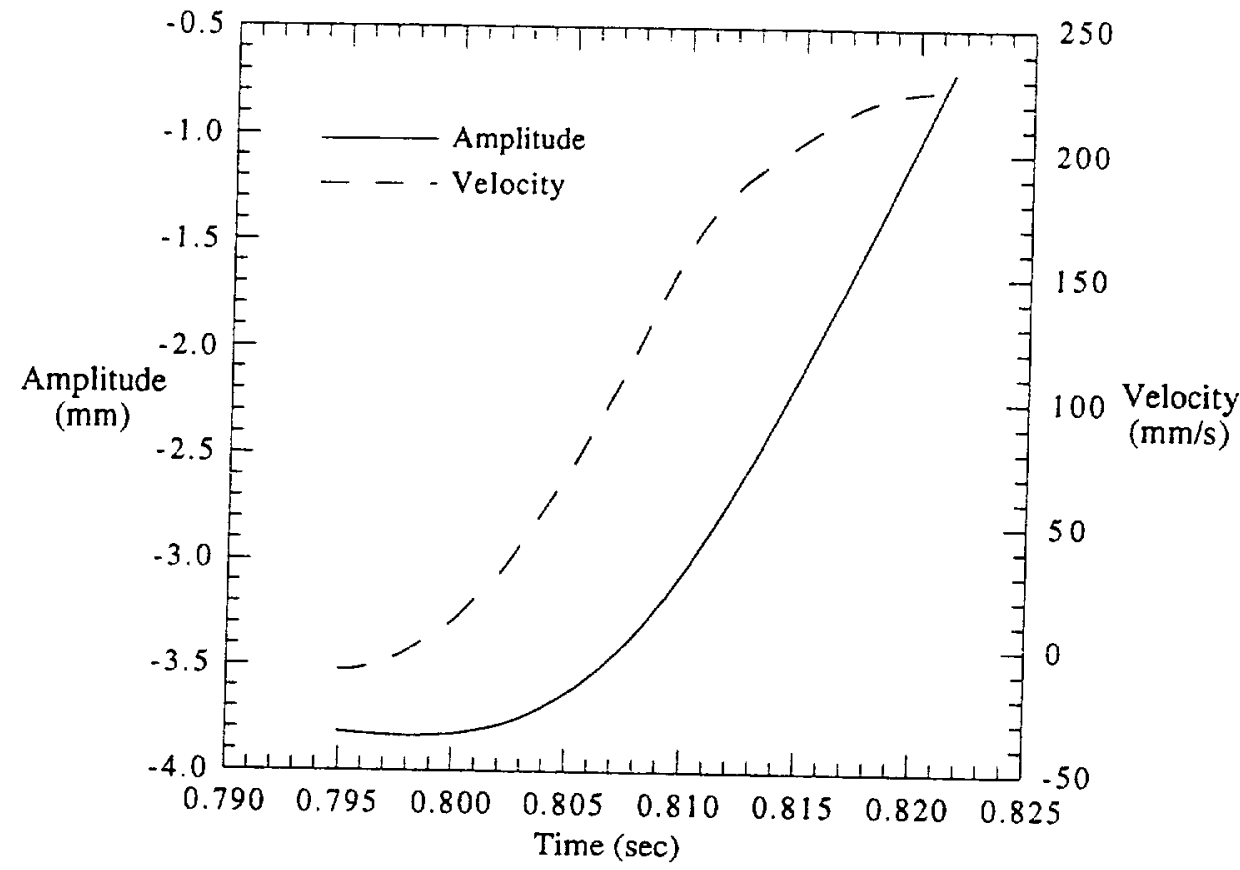
FIGURE 8. A plot of amplitude and velocity versus time obtained from the integration routine for a typical
experiment. 


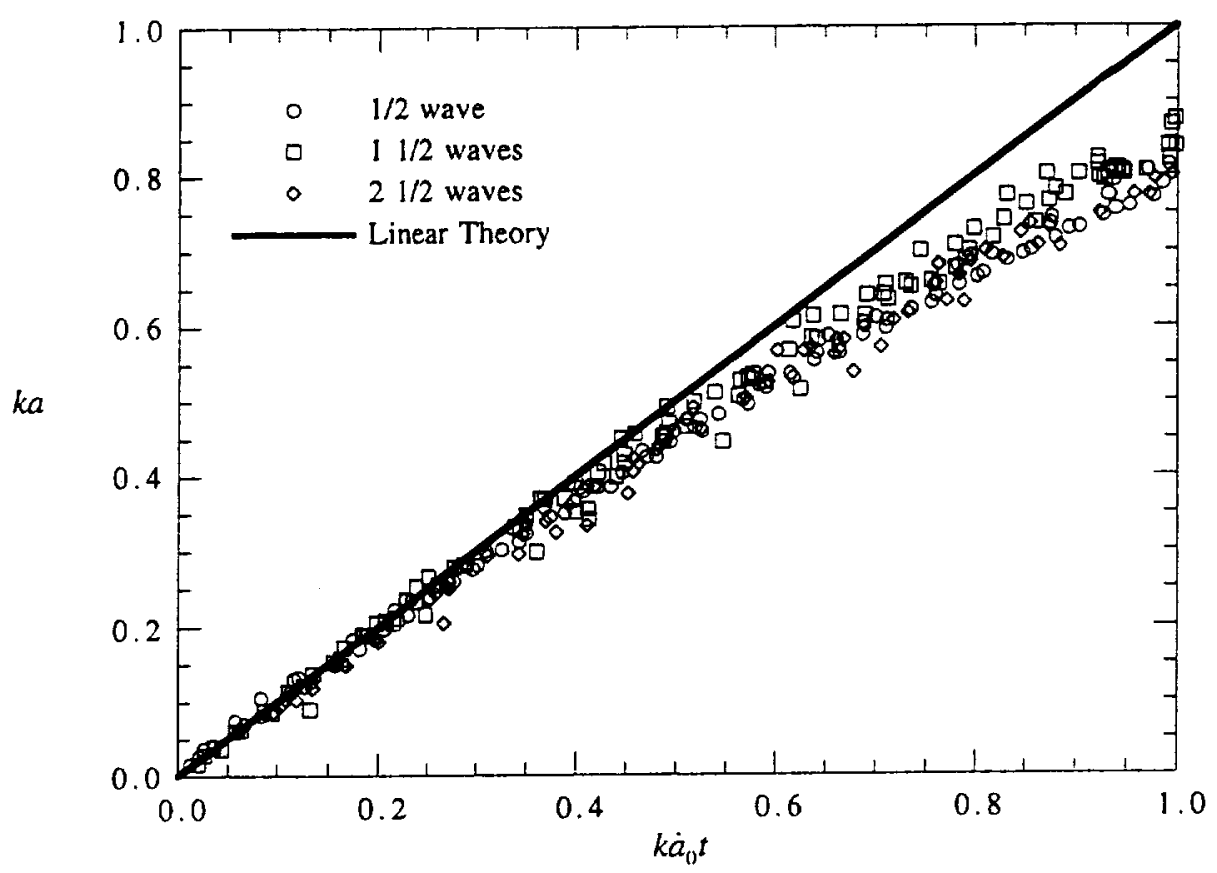

FIGURE 9. Plot of early-time, nondimensional amplitude versus time data along with linear theory. 


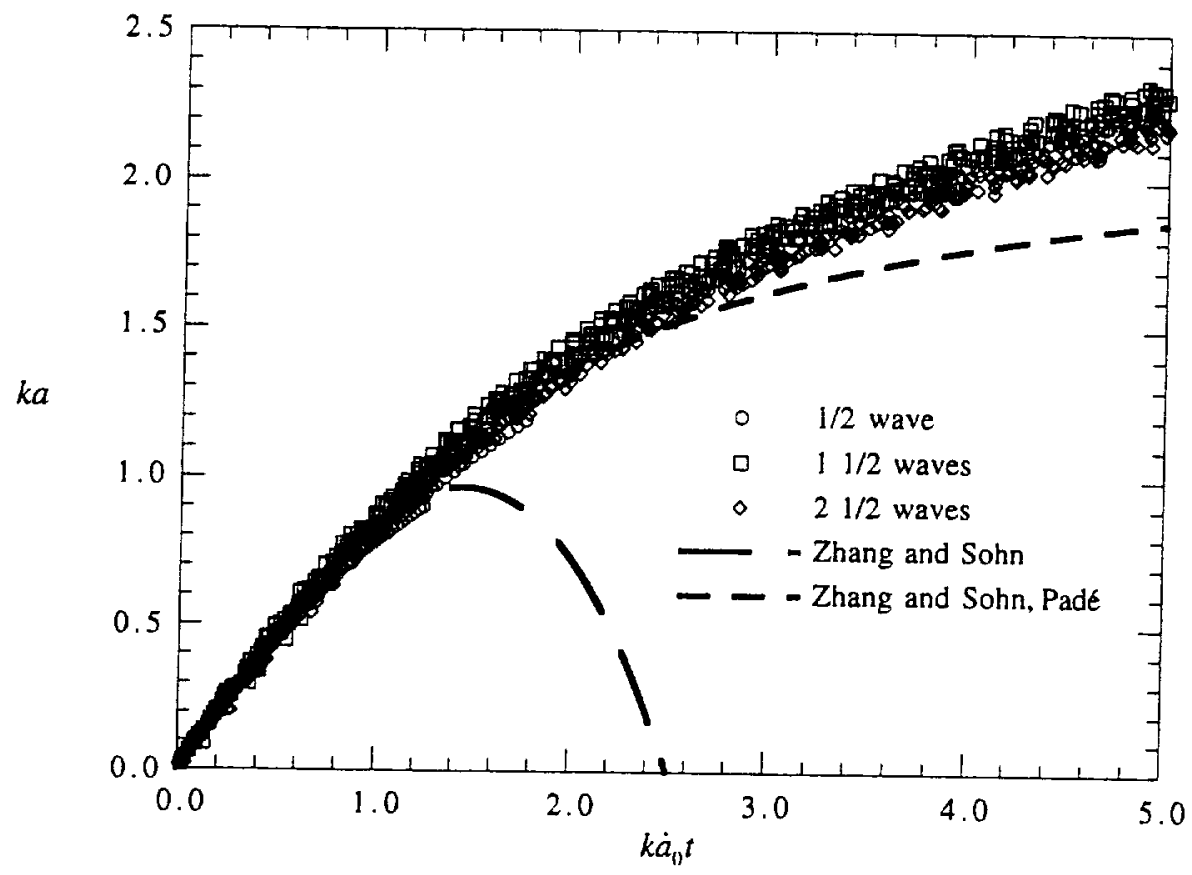

FIGURE 10. Plot of intermediate-time, nondimensional amplitude versus time data along with two curves based on perturbation theories. 


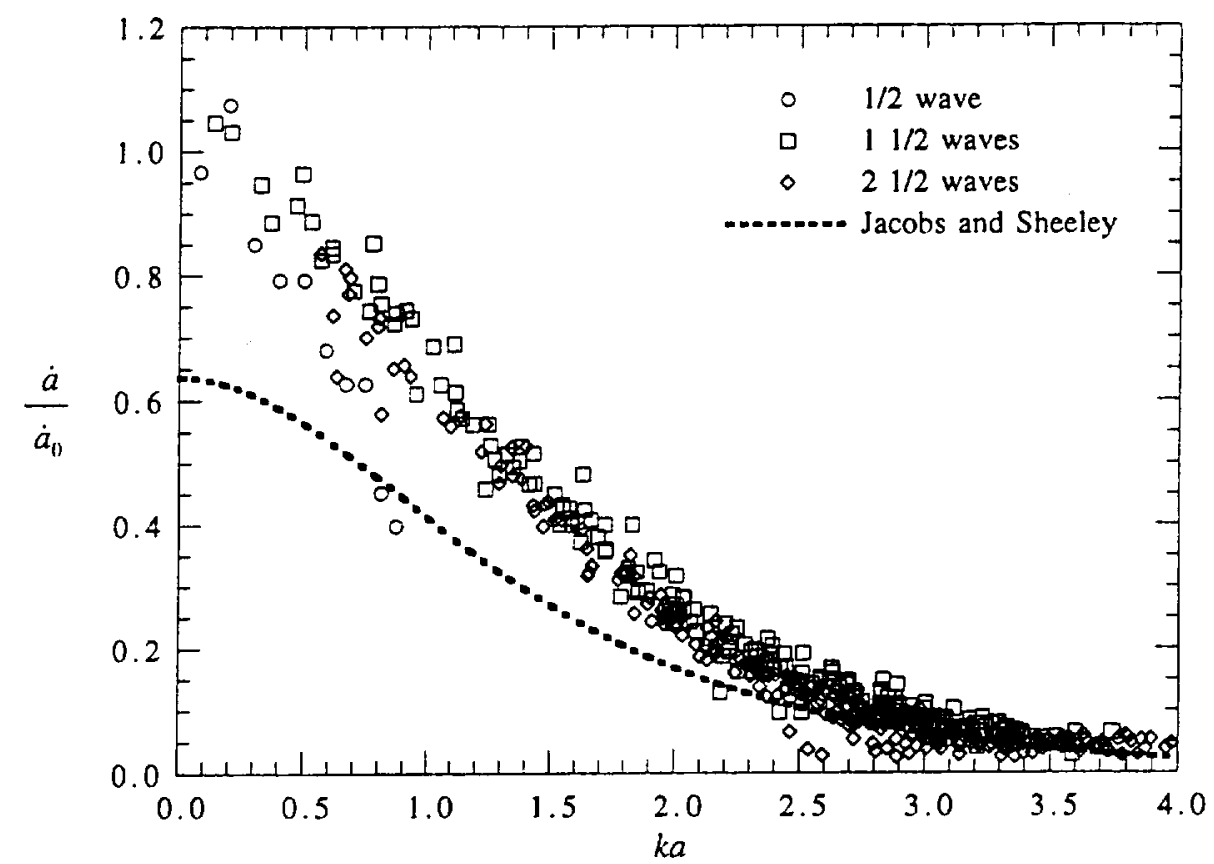

FIGURE 11. Plot of late-time, nondimensional growth rate versus amplitude data along with growth from the vortex model. 


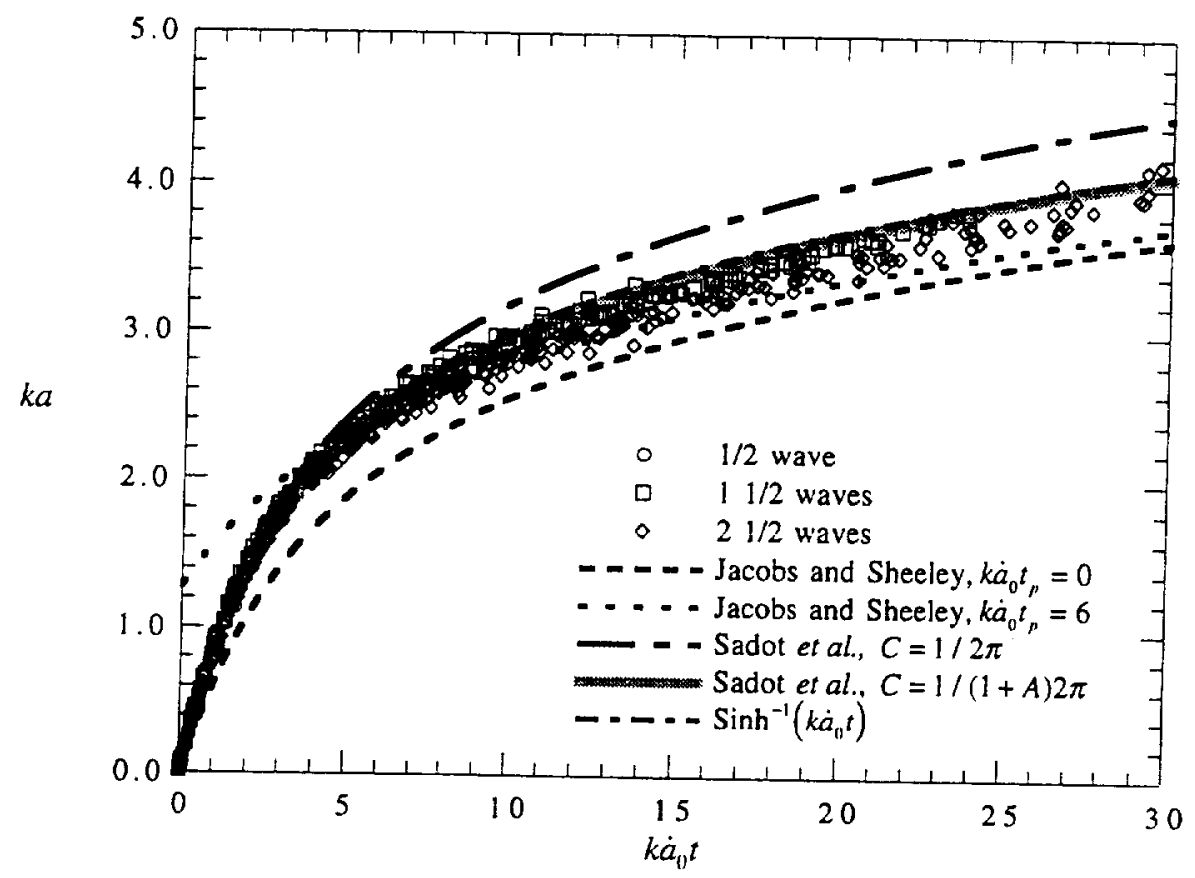
FIGURE 12. Plot of late-time, nondimensional amplitude versus time data along with curves from several
nonlinear theories. 


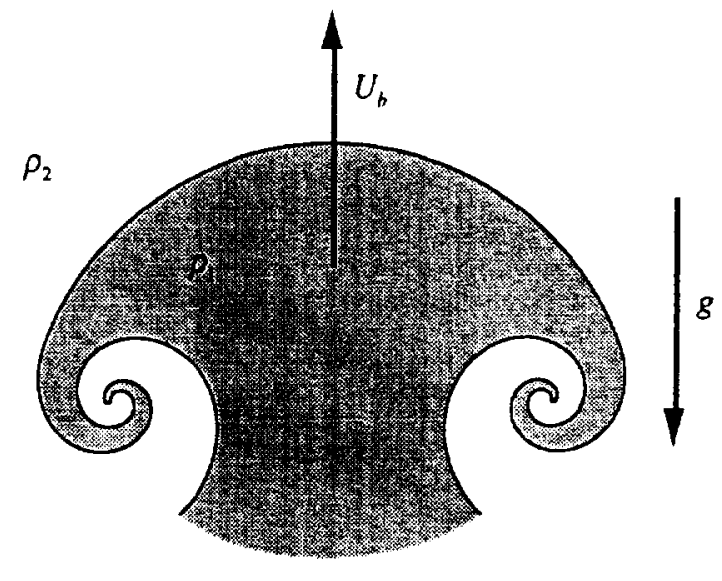

FIGURE 13. Configuration used for the bubble force balance model. 


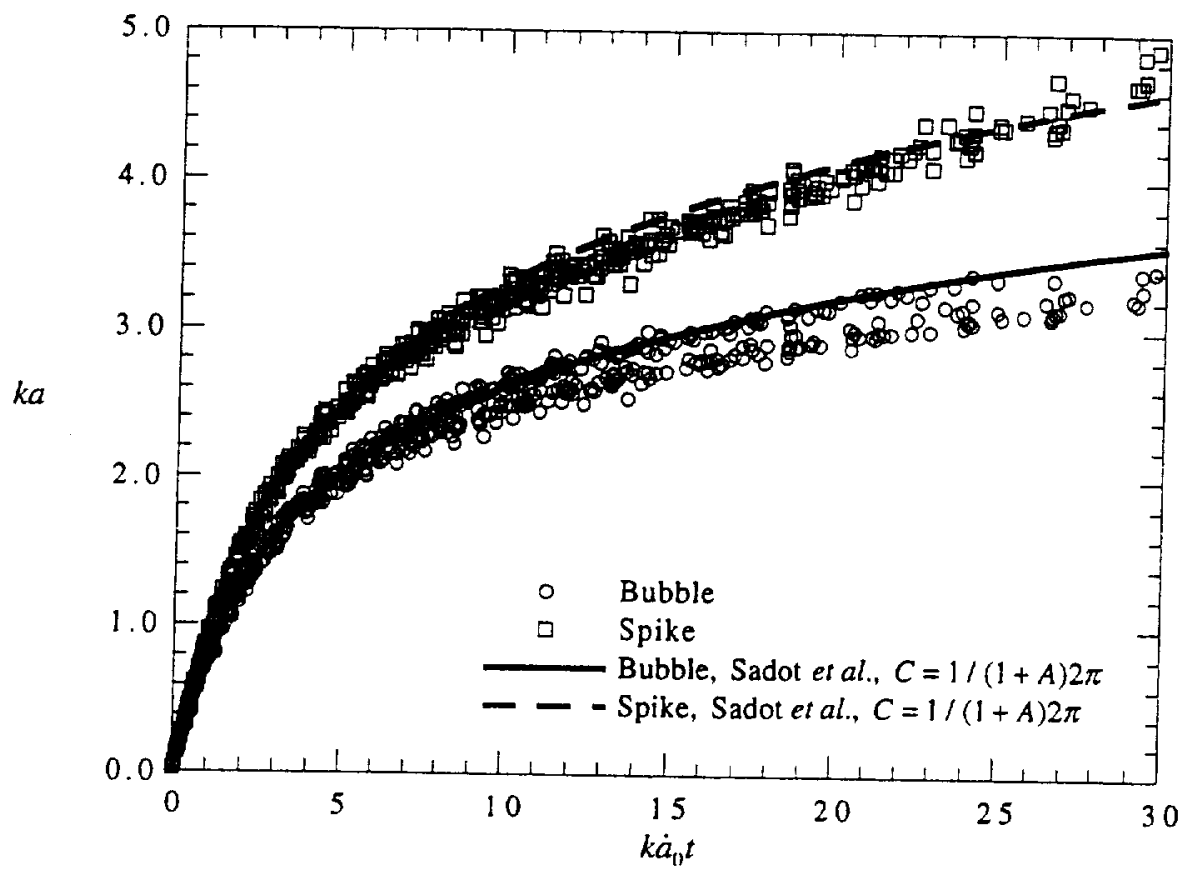

FIGURE 14. Plot of separate bubble and spike amplitudes versus time data along with a theory from Sadot et al. with modified constant $C$. 

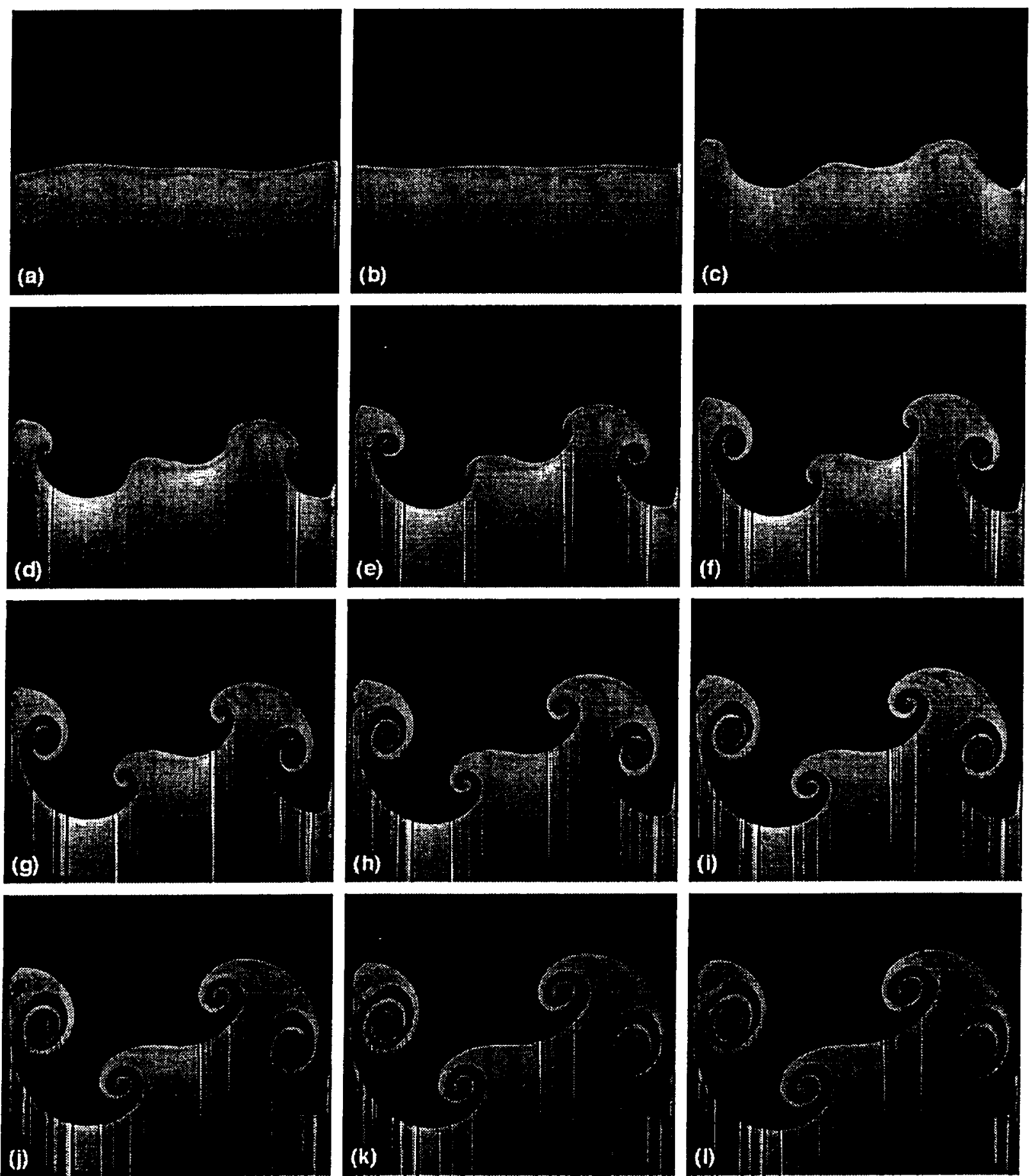

FIGURE 15. A sequence of images from a multi-mode experiment with a combination of $11 / 2$ waves and $21 / 2$ waves as the initial perturbation. Times relative to the midpoint of spring impact are (a) $-15 \mathrm{~ms}$, (b) $18 \mathrm{~ms}$, (c) 101 $\mathrm{ms}$, (d) $185 \mathrm{~ms}$, (e) $285 \mathrm{~ms}$, (f) $368 \mathrm{~ms}$, (g) $452 \mathrm{~ms}$, (h) $552 \mathrm{~ms}$, (i) $635 \mathrm{~ms}$, (j) $719 \mathrm{~ms}$, (k) $819 \mathrm{~ms}$, and (l) $902 \mathrm{~ms}$. 


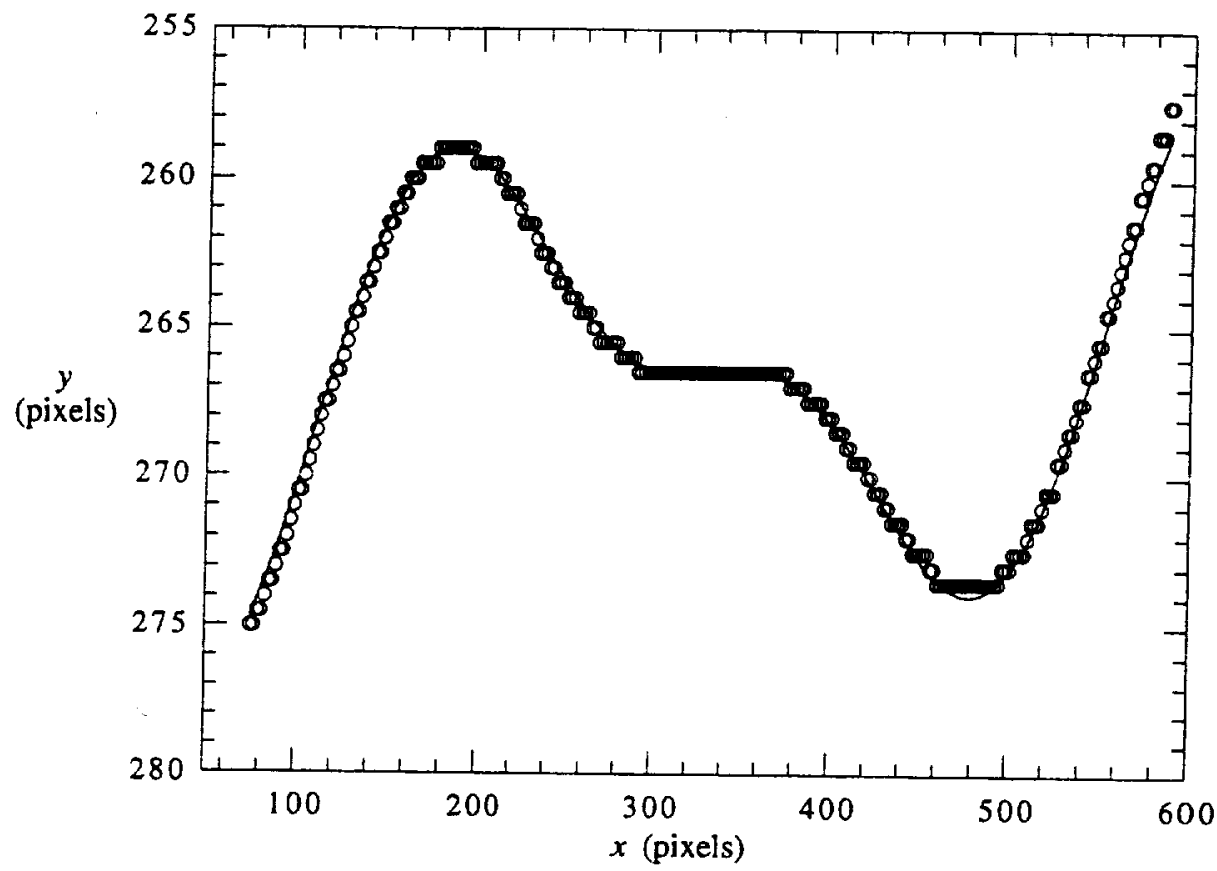

FIGURE 16. Multi-mode sinusoidal curve fit to an edge-detected image from an experiment with a combination of 1 $1 / 2$ and $21 / 2$ waves. 


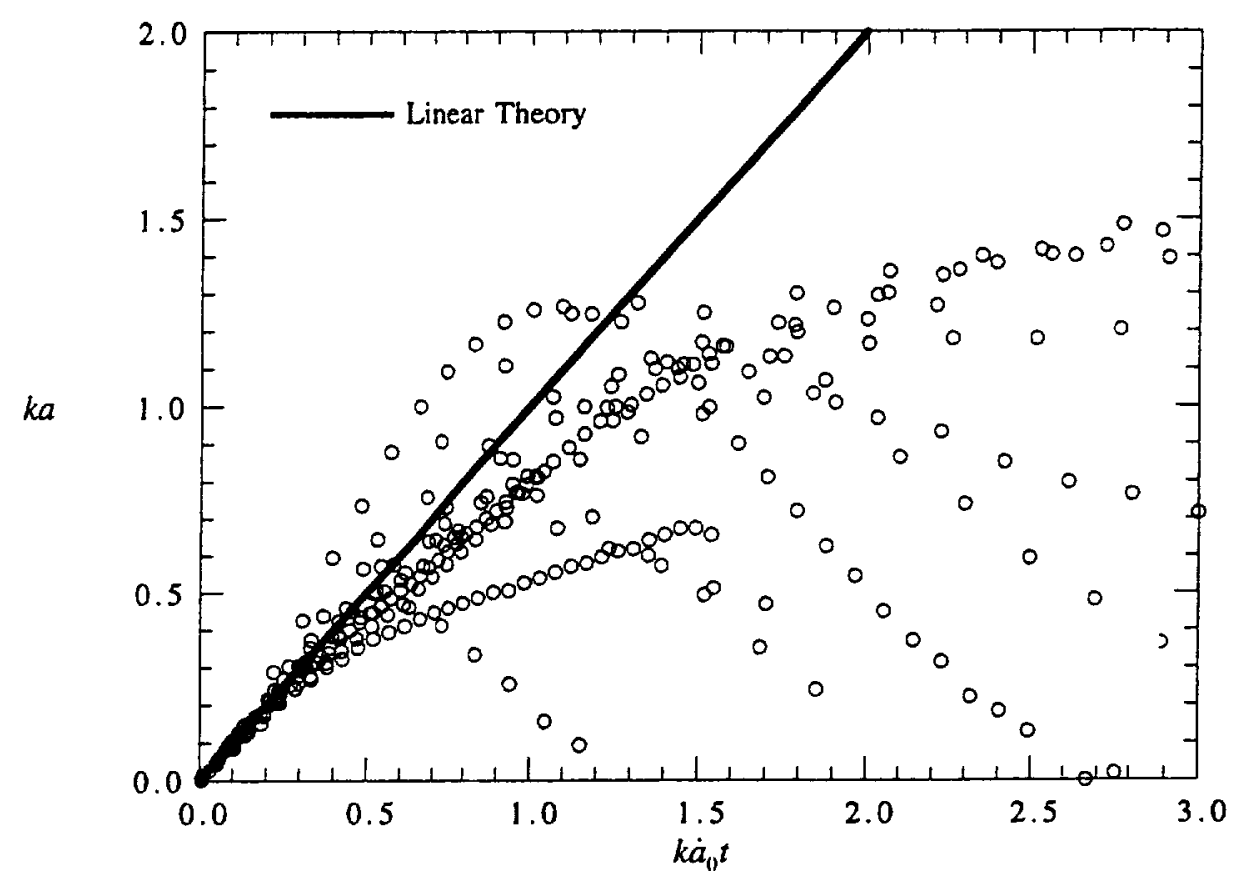

FIGURE 17. Plot of nondimensional amplitude versus time for nine multi-mode experiments with 19 total modes. Mode combinations include: $1 / 2 \& 21 / 2 ; 1 / 2 \& 41 / 2 ; 11 / 2 \& 21 / 2 ; 11 / 2 \& 41 / 2 ; 11 / 2,31 / 2$, \& $41 / 2$; and $21 / 2 \& 41 / 2$. 

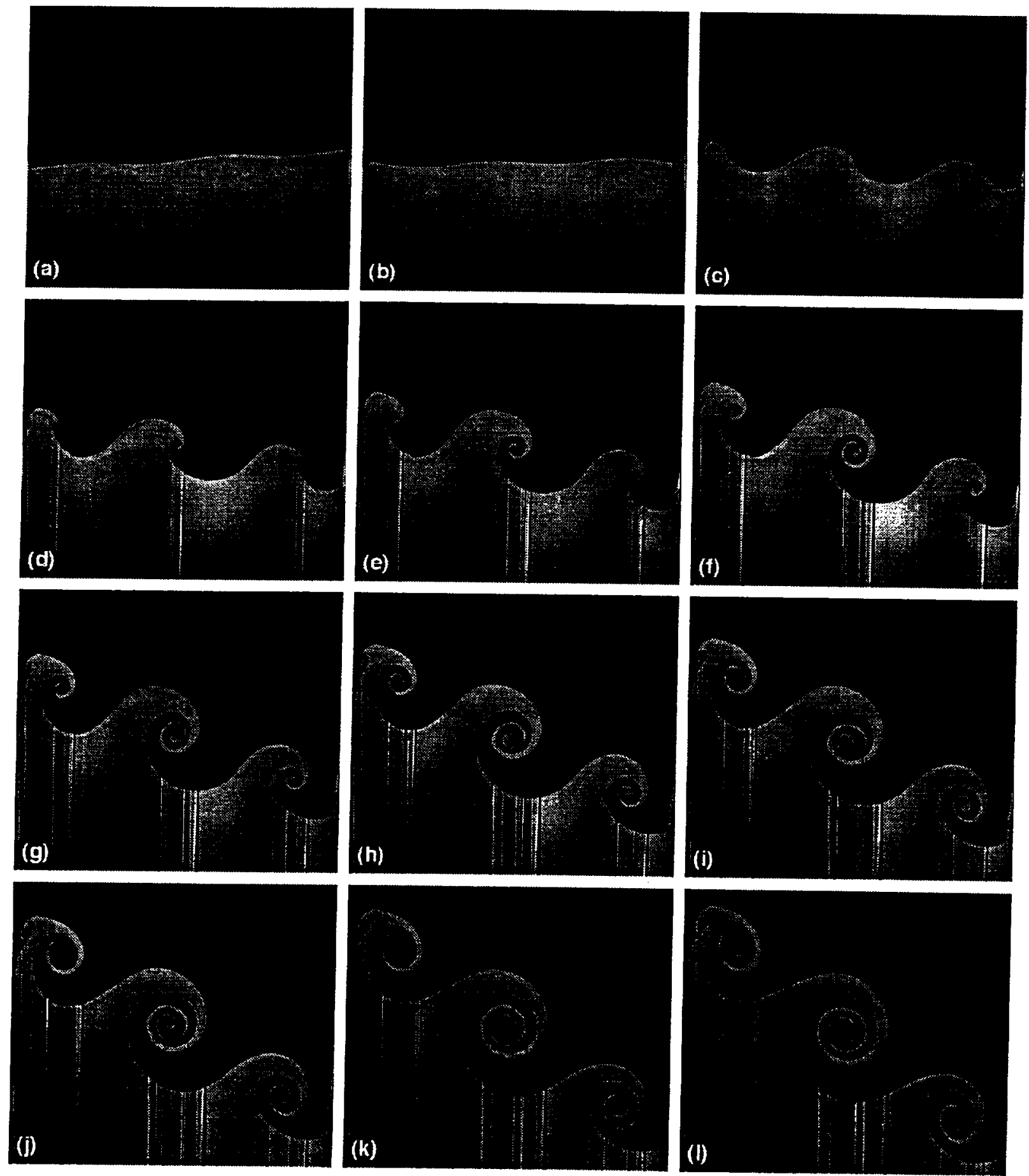

FIGURE 18. A sequence of images from a multi-mode experiment with a combination of a $1 / 2$ wave and $21 / 2$ waves as the initial perturbation. Times relative to the midpoint of spring impact are (a) $-27 \mathrm{~ms}$, (b) $23 \mathrm{~ms}$, (c) $107 \mathrm{~ms}$, (d) $190 \mathrm{~ms}$, (e) $290 \mathrm{~ms}$, (f) $374 \mathrm{~ms}$, (g) $457 \mathrm{~ms}$, (h) $557 \mathrm{~ms}$, (i) $641 \mathrm{~ms}$, (j) $724 \mathrm{~ms}$, (k) $824 \mathrm{~ms}$, and (l) $907 \mathrm{~ms}$. 


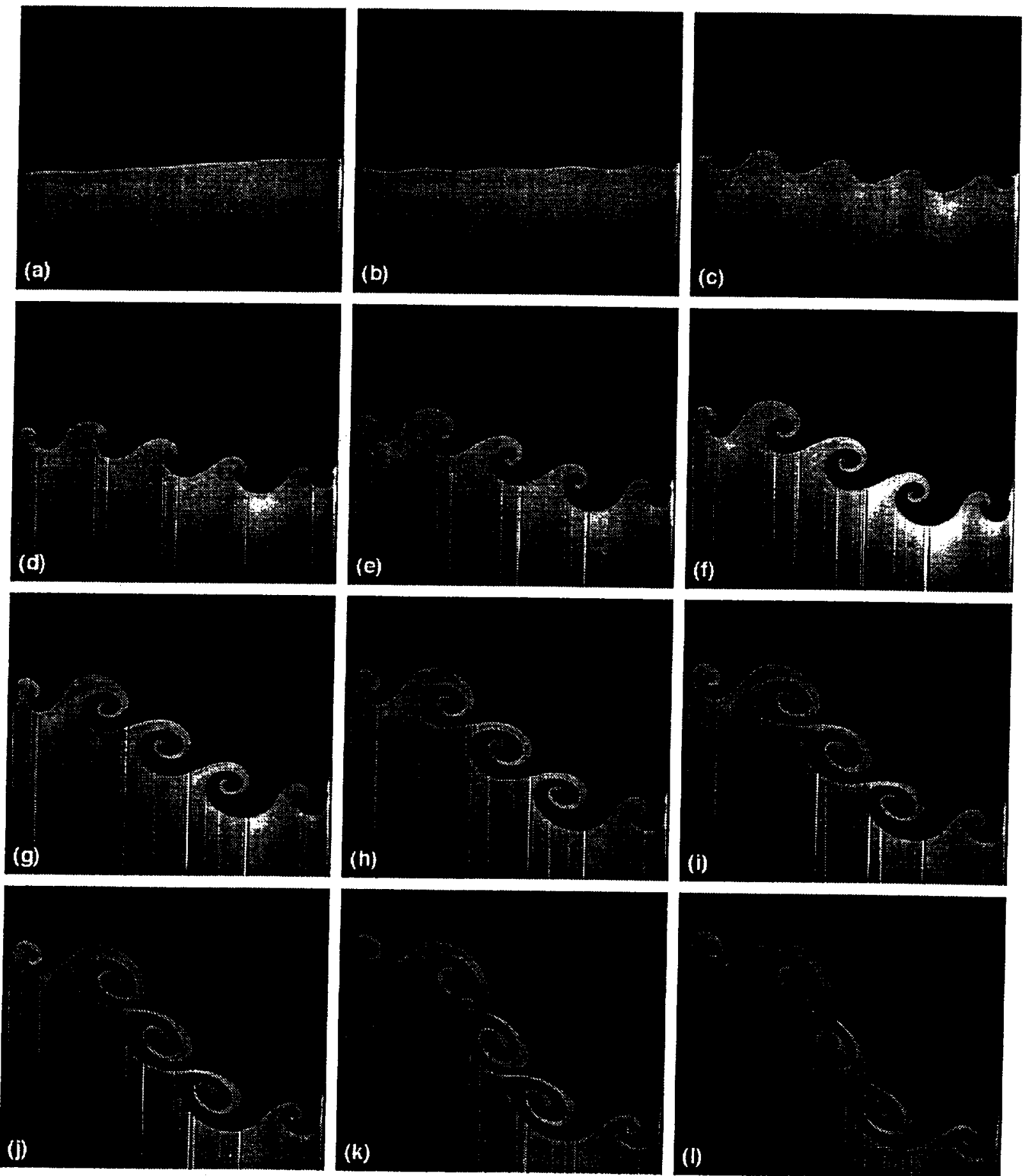

FIGURE 20. A sequence of images from a multi-mode experiment with a combination of a $1 / 2$ wave and $41 / 2$ waves as the initial perturbation. Times relative to the midpoint of spring impact are (a) $-20 \mathrm{~ms}$, (b) $30 \mathrm{~ms}$, (c) $113 \mathrm{~ms}$, (d) $197 \mathrm{~ms}$, (e) $297 \mathrm{~ms}$, (f) $380 \mathrm{~ms}$, (g) $464 \mathrm{~ms}$, (h) $564 \mathrm{~ms}$, (i) $647 \mathrm{~ms}$, (j) $731 \mathrm{~ms}$, (k) $831 \mathrm{~ms}$, and (I) $914 \mathrm{~ms}$. 


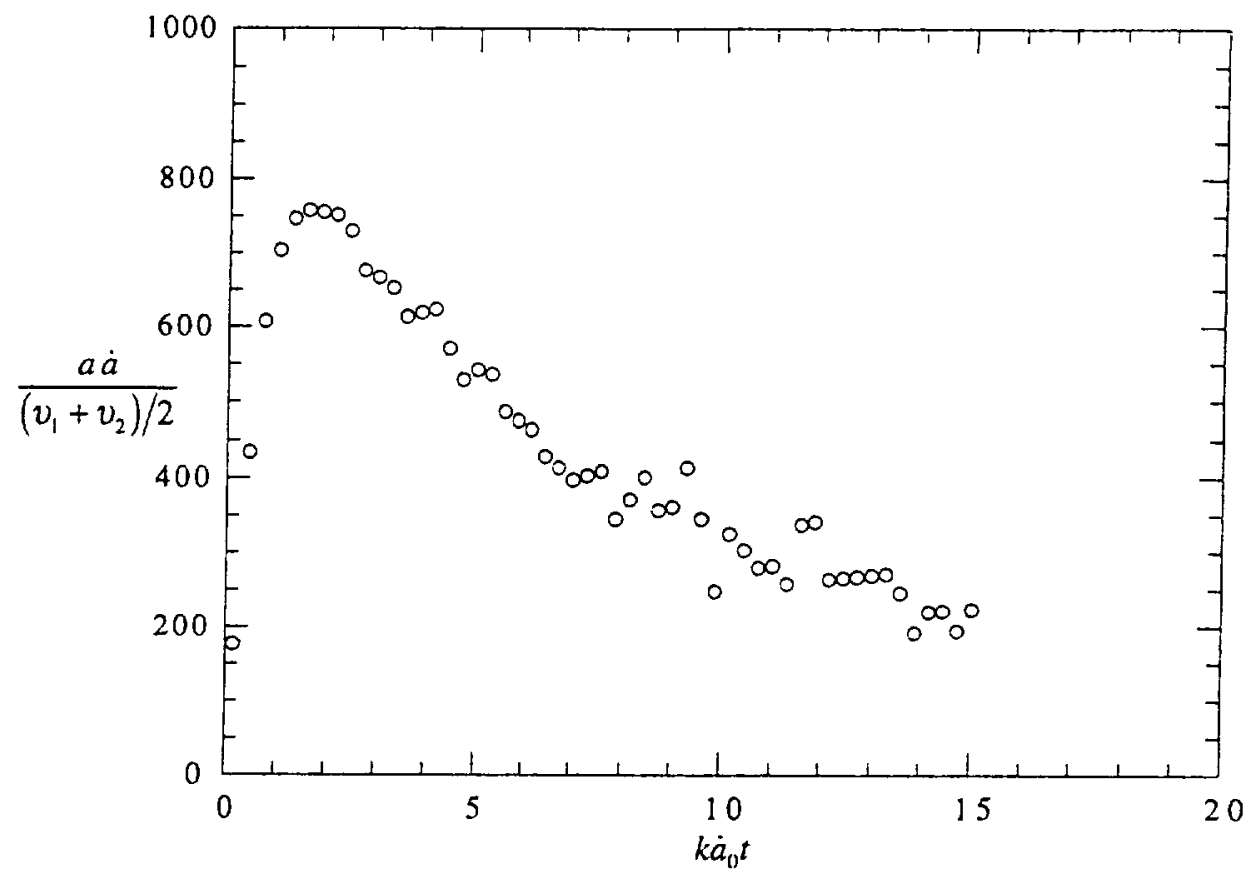

FIGURE 21. Plot of perturbation Reynolds Number (based on amplitude and velocity) versus time for a typical experiment. 


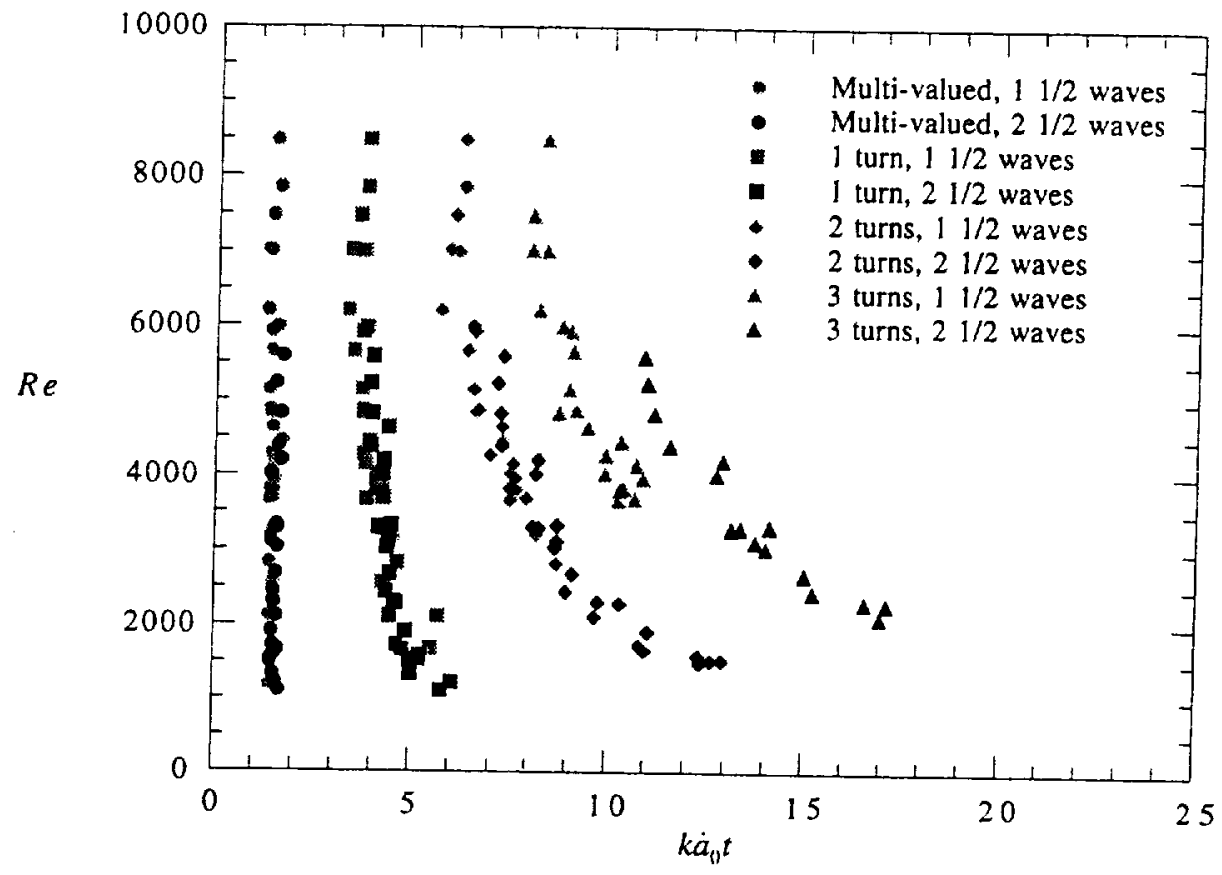

FIGURE 22. Plot of Reynolds Number versus time describing the vortex core evolution. 

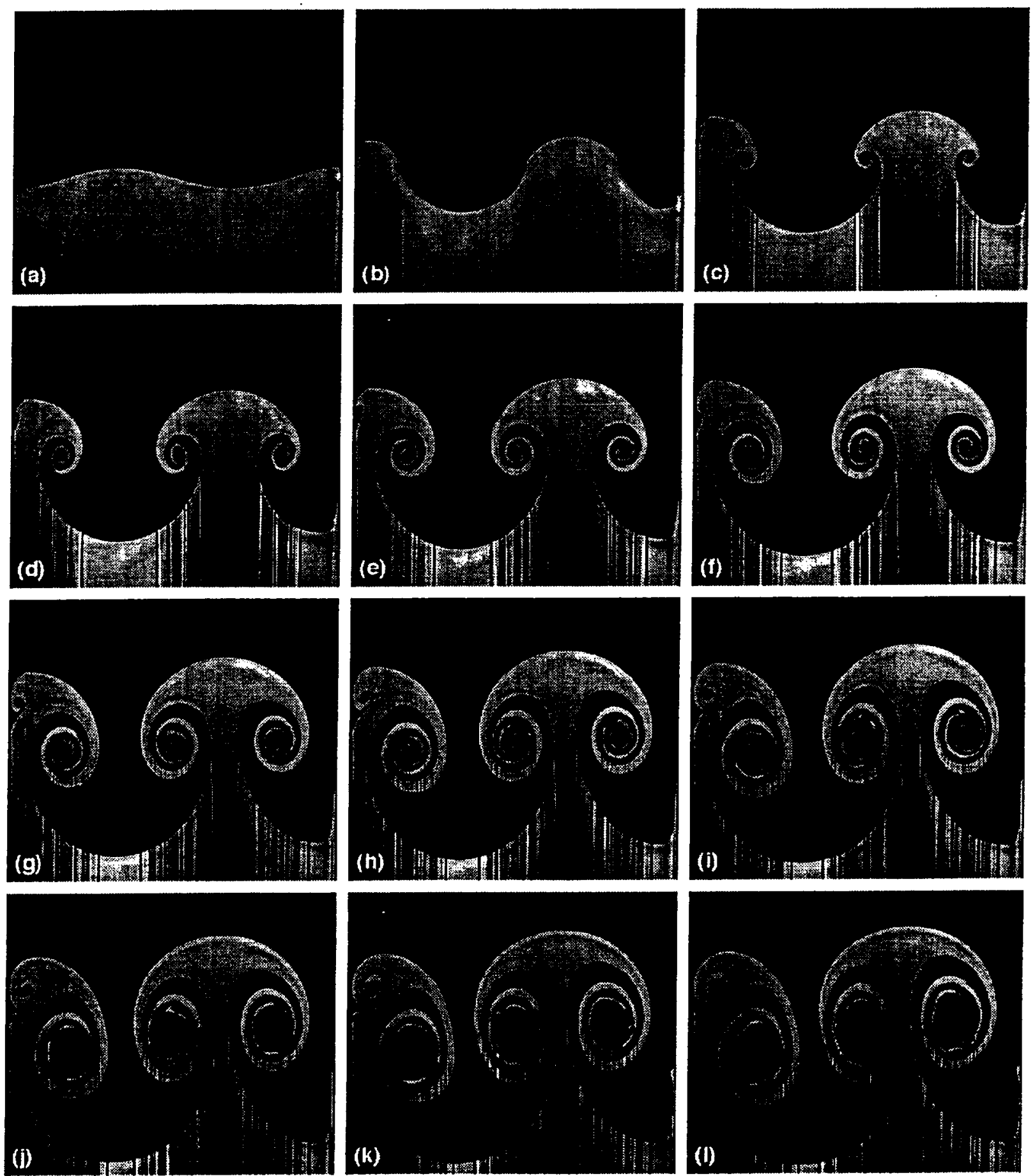

FIGURE 23. A sequence of images from an experiment with $11 / 2$ waves where the vortex core exhibits a secondary instability. Times relative to the midpoint of spring impact are (a) $-24 \mathrm{~ms}$, (b) $93 \mathrm{~ms}$, (c) $176 \mathrm{~ms}$, (d) $260 \mathrm{~ms}$, (e) $343 \mathrm{~ms}$, (f) $426 \mathrm{~ms}$, (g) $510 \mathrm{~ms}$, (h) $593 \mathrm{~ms}$, (i) $677 \mathrm{~ms}$, (j) $760 \mathrm{~ms}$, (k) $843 \mathrm{~ms}$, and (l) $910 \mathrm{~ms}$. 


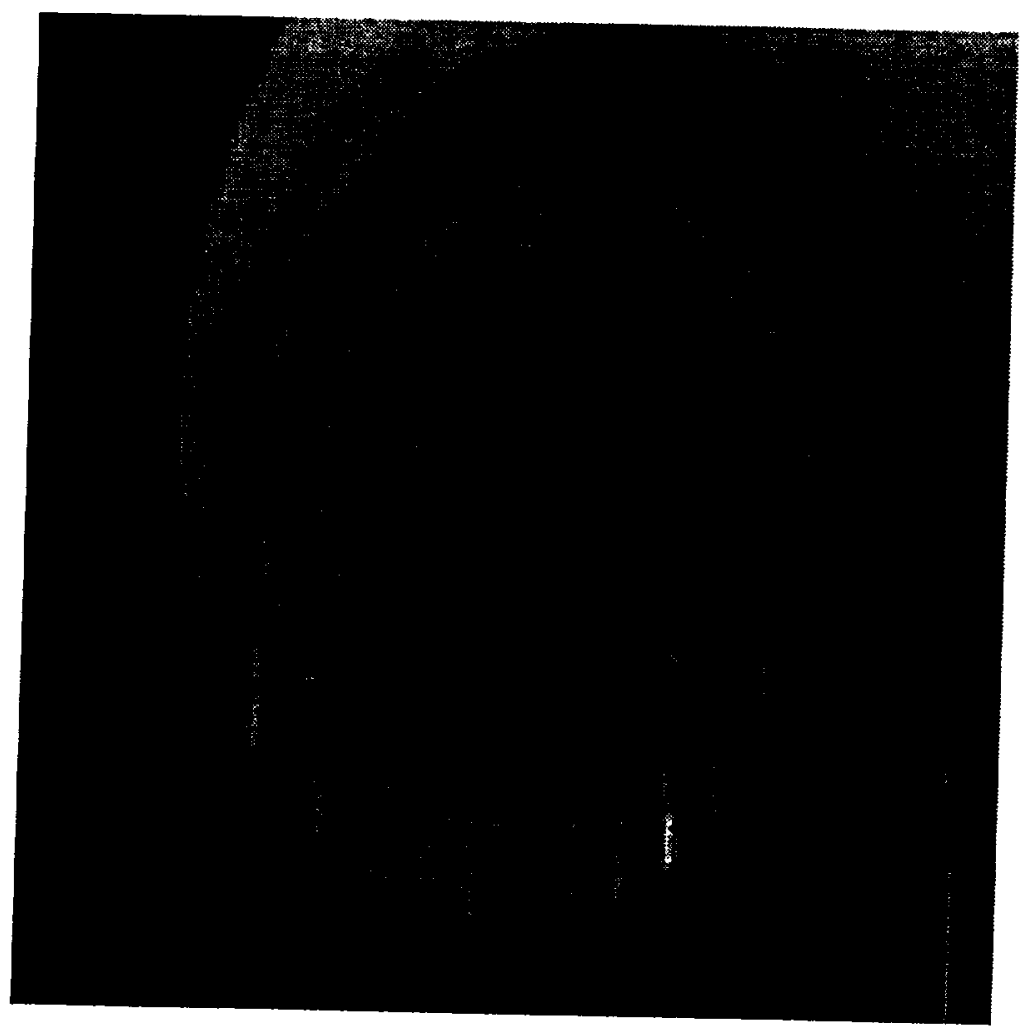

FIGURE 24. Close-up of a vortex core during the early stages of a secondary instability. 


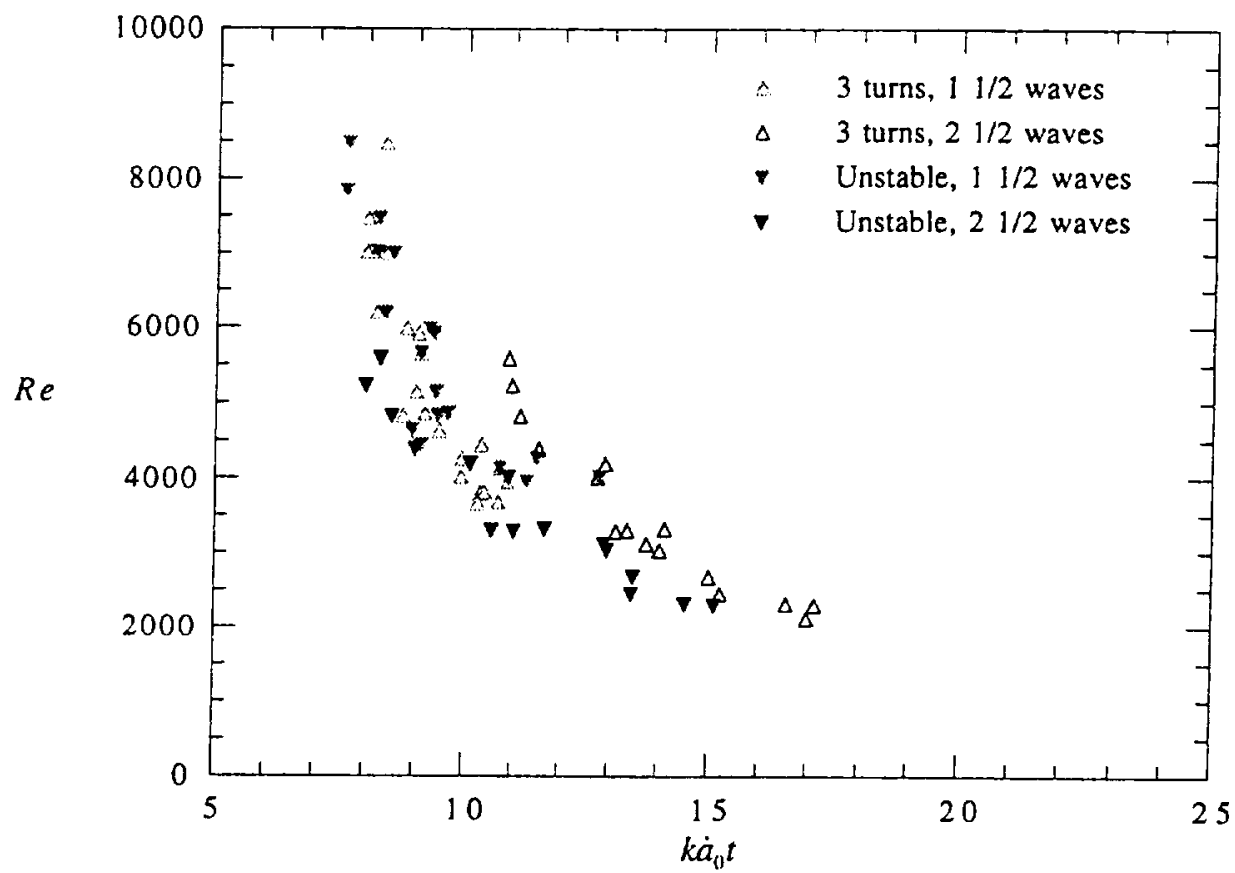

FIGURE 25. Plot of Reynolds Number versus time for the vortex core to become unstable. Also shown is the time for the vortex to complete 3 turns. 
